\title{
Implementation of the full viscoresistive magnetohydrodynamic equations in a nonlinear finite element code
}

\author{
J.W. Haverkort ${ }^{\mathrm{a}, \mathrm{b}}$, H.J. de Blank ${ }^{\text {b }}$, G.T.A. Huysmans ${ }^{\mathrm{c}}$, J. Pratt ${ }^{\mathrm{b}}$, B. Koren ${ }^{\mathrm{d}, *}$ \\ a Centrum Wiskunde E' Informatica, P.O. Box 94079, 1090 GB Amsterdam, The Netherlands \\ b Dutch Institute for Fundamental Energy Research, P.O. Box 6336, 5600 HH Eindhoven, The Netherlands \\ c ITER Organization, Route de Vinon sur Verdon, 13115 Saint Paul Lez Durance, France \\ d Eindhoven University of Technology, P.O. Box 513, 5600 MB Eindhoven, The Netherlands
}

\section{A R T I C L E I N F O}

\section{Article history:}

Received 4 December 2015

Received in revised form 2 April 2016

Accepted 2 April 2016

Available online 12 April 2016

\section{Keywords:}

Magnetohydrodynamics

Finite element method

Implicit time integration

Magnetic vector potential

Anisotropic diffusion

Internal kink mode

Tearing mode

Ballooning mode

\begin{abstract}
A B S T R A C T
Numerical simulations form an indispensable tool to understand the behavior of a hot plasma that is created inside a tokamak for providing nuclear fusion energy. Various aspects of tokamak plasmas have been successfully studied through the reduced magnetohydrodynamic (MHD) model. The need for more complete modeling through the full MHD equations is addressed here. Our computational method is presented along with measures against possible problems regarding pollution, stability, and regularity.

The problem of ensuring continuity of solutions in the center of a polar grid is addressed in the context of a finite element discretization of the full MHD equations. A rigorous and generally applicable solution is proposed here.

Useful analytical test cases are devised to verify the correct implementation of the momentum and induction equation, the hyperdiffusive terms, and the accuracy with which highly anisotropic diffusion can be simulated. A striking observation is that highly anisotropic diffusion can be treated with the same order of accuracy as isotropic diffusion, even on non-aligned grids, as long as these grids are generated with sufficient care. This property is shown to be associated with our use of a magnetic vector potential to describe the magnetic field.

Several well-known instabilities are simulated to demonstrate the capabilities of the new method. The linear growth rate of an internal kink mode and a tearing mode are benchmarked against the results of a linear MHD code. The evolution of a tearing mode and the resulting magnetic islands are simulated well into the nonlinear regime. The results are compared with predictions from the reduced MHD model.

Finally, a simulation of a ballooning mode illustrates the possibility to use our method as an ideal MHD method without the need to add any physical dissipation.
\end{abstract}

(c) 2016 Elsevier Inc. All rights reserved.

\footnotetext{
* Corresponding author.

E-mail address: b.koren@tue.nl (B. Koren).
} 


\section{Introduction}

The hot plasma in a tokamak knows many ways to escape the grip of the confining magnetic field. Most of these have been long understood and this understanding has led to a tremendous increase in the time a plasma can be kept in place. A first understanding of the development of instabilities in tokamak plasmas starts with an understanding of their linear phase. The theory of magnetohydrodynamics (MHD) has been successful in understanding many linear instabilities like kink, tearing, and ballooning modes. The kink mode is at the basis of what is called the sawtooth instability, named after the shape of the resulting magnetic signals. Tearing modes grow to form magnetic islands, while the ballooning mode plays an important role in the formation of Edge Localized Modes (ELMs). An accurate description of these phenomena typically requires the inclusion of effects outside of the MHD model, like the effects of fast particles or neoclassical effects. Linear MHD instabilities and their nonlinear development can already account for many aspects of the observations though.

While purely theoretical analyses are arguably still the most important tool for understanding the linear phase of instabilities, for their nonlinear development this role is more and more taken over by numerical simulations. Over the last few decades the computing power has increased to a level that the nonlinear development of instabilities can be routinely simulated with sufficient resolution in both space and time. As a result, many codes have been developed that can solve a subor super-set of the MHD equations. Codes used for tokamak applications include NIMROD [1,2], BOUT++ [3,4], M3D(-C $\left.{ }^{1}\right)$ $[5,6]$, XTOR(-2F) [7,8], FAR [9], MEGA [10], and JOREK.

The JOREK code was initially developed as a reduced MHD code. It has been used to calculate various instabilities like the peeling(-tearing) mode [11], ballooning modes [12], and their combined nonlinear development in the form of ELMs [13-16]. The influence of flows on ELMs [13,17] and various mechanisms to trigger or mitigate instabilities through resonant magnetic perturbations [18,19], pellet injection [14], and massive gas injection [20] have also been studied extensively with JOREK.

These simulations were all performed using the reduced MHD model. In this model typically only one or two variables are used to describe the velocity. The primary difference with the full MHD model is the possibility of supporting fast magneto-acoustic waves, which require compression of the toroidal magnetic field. Since these waves are very stable and have a frequency that is much higher than most other phenomena of interest, this is typically considered to be an advantage. However, there are situations in which compression of the toroidal magnetic field can be important. A particular instance may be the accurate description of fast poloidal rotation, for which 'magnetic pumping' acts as a damping mechanism. Since in this case anisotropic pressure and diamagnetic effects are expected to be important as well, extension from reduced to full MHD can be considered as an important step towards more complete tokamak modeling.

The numerical methods for the reduced MHD equations, as implemented in the JOREK code, are described in [21,22]. Here we focus on the extension of these methods to the full MHD equations. In Section 2 we first describe the strong form of the equations and how the gauge freedom introduced by the use of a magnetic vector potential was used. Next, after discussing the simple boundary conditions used in this work, we derive a suitable weak form of the equations.

Some background with respect to the numerics is presented in Section 3. The spatial discretization is discussed as far as required for what follows, while the time integration is discussed in more detail. In Section 4 we discuss our solution to a problem encountered with spectral pollution.

Next, we thoroughly verify our implementation through a series of tests. We test the equations individually in Section 5 and the complete system of equations in Section 6. Finally, Appendix A discusses a possible method to guarantee continuity of the solutions at the center of a polar grid.

\section{Model}

\subsection{Equations}

Our goal is to implement the following system of viscoresistive MHD equations:

$$
\begin{aligned}
\frac{\partial \rho}{\partial t} & =-\nabla \cdot\left(\rho \mathbf{u}+\mathbf{q}_{m}\right)+S_{m}, \\
\frac{\partial \rho \mathbf{u}}{\partial t} & =-\mathbf{u} \nabla \cdot(\rho \mathbf{u})-\rho \mathbf{u} \cdot \nabla \mathbf{u}-\nabla p+\mathbf{J} \times \mathbf{B}+\nabla \cdot \mathbf{T}_{v}, \\
\frac{\partial p}{\partial t} & =-\nabla \cdot\left[\gamma p \mathbf{u}+(\gamma-1) \mathbf{q}_{h}\right]+(\gamma-1)\left(\mathbf{u} \cdot \nabla p+\eta J^{2}+\mathbf{T}_{v}: \nabla \mathbf{u}+S_{h}\right), \\
\frac{\partial \mathbf{A}}{\partial t} & =-\eta \mathbf{J}+\mathbf{u} \times \mathbf{B},
\end{aligned}
$$

for the primitive variables $\rho, T, \mathbf{u}$ and $\mathbf{A}$, the density, temperature, velocity, and magnetic vector potential respectively. The coefficient $\gamma$ is the adiabatic constant expressing the ratio of specific heats. In this work we will use the mono-atomic ideal gas value $\gamma=5 / 3$. For describing other plasma phenomena different values may however be more appropriate [23]. As secondary variables we use the current density $\mathbf{J}=\nabla \times \mathbf{B}$, and pressure $p=\rho T$. The magnetic field $\mathbf{B}$ is expressed in terms of a magnetic vector potential $\mathbf{A}$ as 


$$
\mathbf{B}=\nabla \times \mathbf{A}+\mathbf{F},
$$

where we allow for the addition of a divergence-free vector $\mathbf{F}$ that is constant in time, see section 2.8 . The continuity and heat equation are supplemented with mass and heat sources $S_{m}$ and $S_{h}$. The constitutive relations for the fluxes $\mathbf{q}_{m}$ and $\mathbf{q}_{h}$ are given by

$$
\begin{aligned}
& \mathbf{q}_{m}=-\left(D_{\perp} \nabla_{\perp} \rho+D_{\|} \nabla_{\|} \rho\right)+D_{h} \nabla \nabla^{2} \rho, \\
& \mathbf{q}_{h}=-\left(K_{\perp} \nabla_{\perp} T+K_{\|} \nabla_{\|} T\right),
\end{aligned}
$$

where $\nabla_{\|}=\left(\mathbf{B} / B^{2}\right) \mathbf{B} \cdot \nabla$ and $\nabla_{\perp}=\nabla-\nabla_{\|}$. The parallel conductivity $K_{\|}$and mass diffusion coefficient $D_{\|}$in general depend on the temperature, which is fully allowed for in the code, but will not be done in the test cases to be considered in this paper. The perpendicular diffusion coefficients $D_{\perp}$ and $K_{\perp}$ and the hyperdiffusion coefficient $D_{h}$ can be used to phenomenologically model turbulence effects beyond MHD, which can be useful for simulations on a transport time scale. Hyperdiffusive terms may also be introduced in the temperature equation, or the induction equation, as in [4,24].

The righthand side of the momentum equation (1b) can be written as the divergence of the stress tensor

$$
\mathbf{T}=-\left(p+\frac{1}{2} B^{2}\right) \mathbf{g}-\rho \mathbf{u u}+\mathbf{B B}+\mathbf{T}_{v}
$$

Under the assumptions of isotropy and a linear relation between stress and strain, the most general viscous stress tensor $\mathbf{T}_{v}$ is given by

$$
\begin{gathered}
\mathbf{T}_{v}=\mathbf{T}_{N}+\lambda(\nabla \cdot \mathbf{u}) \mathbf{g}, \\
\mathbf{T}_{N}=\mu\left(\nabla \mathbf{u}+(\nabla \mathbf{u})^{T}\right) .
\end{gathered}
$$

Here $\mathbf{T}_{N}$ is the Newtonian part of the stress tensor proportional to the dynamic viscosity $\mu, \mathbf{g}$ is the metric tensor, and $\lambda$ the second coefficient of viscosity. The quantity $\mu_{B}=\lambda+2 \mu / 3$ is called the 'bulk viscosity' or 'dilatational viscosity' and is, confusingly, also sometimes used in place of $\lambda$ in Eq. (5a). Throughout this paper we use $\lambda=\mu=0$, except in a test case shortly discussed in Section 5.1 where we use a very large value of $\lambda$ to enforce incompressibility.

\subsection{Gauge}

Eq. (1d) for the magnetic field, results from 'uncurling' Faraday's law:

$$
\frac{\partial \mathbf{B}}{\partial t}=-\nabla \times \mathbf{E}
$$

Using Eq. (2) with $\partial \mathbf{F} / \partial t=0$ gives

$$
\frac{\partial \mathbf{A}}{\partial t}=-\mathbf{E} .
$$

Eq. (1d) is obtained after using Ohm's law for moving media:

$$
\mathbf{J}=\eta^{-1}(\mathbf{E}+\mathbf{u} \times \mathbf{B}),
$$

where $\mathbf{E}$ is the electric field. To Eq. (7) the gradient of an arbitrary time-dependent scalar field $\Phi$ can be added, without changing Eq. (6). We have used this 'gauge freedom' to set $\Phi=0$, with obvious advantages. This gauge is called the Weyl gauge, also known as the Hamiltonian or temporal gauge. In the Weyl gauge, a time-independent gradient of a scalar field can still be added. Therefore the gauge is not completely fixed, which is sometimes referred to as an 'incomplete gauge'. An electric potential $\Phi$ can always be reintroduced by replacing a part of $\partial \mathbf{A} / \partial t$ with $\nabla \Phi$. This gauge transformation leaves both the electric and magnetic fields unchanged.

Several other choices for $\Phi$, like the Coulomb gauge in which $\nabla \cdot \mathbf{A}=0$, require the solution of a costly Poisson equation. Some numerical aspects of the various gauges in a finite volume setting are described in [25]. An interesting option specifically for tokamak applications is presented in [6], where instead of an additional variable $\Phi$ to fix the gauge, one variable less is used. The freedom in $\mathbf{A}$ introduced by $\nabla \cdot \mathbf{B}=0$ is used to describe the magnetic vector potential in terms of only two scalar components instead of three.

\subsection{Weak forms}

To obtain the weak formulation of the equations (1a)-(1d) we multiply these with a test function and integrate over the entire domain. We then have the choice of partially integrating some or all of the terms. This choice potentially influences stability and accuracy. Also, a smart choice of which terms to partially integrate can lead to a more convenient implementation of the equations. 


\subsubsection{Continuity equation}

We multiply Eq. (1a) for the density $\rho$ with a test function $\rho^{*}$ and integrate over the entire domain

$$
\begin{aligned}
\int \rho^{*} \frac{\partial \rho}{\partial t} d V & =\int\left[-\rho^{*} \nabla \cdot\left(\rho \mathbf{u}+\mathbf{q}_{m}\right)+\rho^{*} S_{m}\right] d V \\
& =\int\left[\left(\rho \mathbf{u}+\mathbf{q}_{m}\right) \cdot \nabla \rho^{*}+\rho^{*} S_{m}\right] d V-\oint \rho^{*}\left(\rho \mathbf{u}+\mathbf{q}_{m}\right) \cdot d \mathbf{S},
\end{aligned}
$$

where the surface element $d \mathbf{S}$ is oriented normal to the boundary in the outward direction. The weak formulation of the pressure equation (1c) follows in a similar way.

For the hyperdiffusive term $-D_{h} \nabla \nabla^{2} \rho$ in $\mathbf{q}_{m}$ we can perform one more partial integration to write ${ }^{1}$

$$
-\int D_{h} \nabla \nabla^{2} \rho \cdot \nabla \rho^{*} d V=\int \nabla^{2} \rho \nabla \cdot\left(D_{h} \nabla \rho^{*}\right) d V-\int D_{h} \nabla^{2} \rho \nabla \rho^{*} \cdot d \mathbf{S} .
$$

\subsubsection{Momentum equation}

Taking the inner product of Eq. (1b) with a vectorial test function $\mathbf{m}^{*}$ and integrating by parts we get

$$
\int \mathbf{m}^{*} \cdot \frac{\partial \rho \mathbf{u}}{\partial t} d V=\int-\mathbf{T}: \nabla \mathbf{m}^{*} d V+\int \mathbf{T} \cdot \mathbf{m}^{*} \cdot d \mathbf{S} .
$$

For the stress tensor (4) the boundary term reads

$$
\int \mathbf{T} \cdot \mathbf{m}^{*} \cdot d \mathbf{S}=\int\left[-\left(p+\frac{1}{2} B^{2}-\lambda(\nabla \cdot \mathbf{u})\right) \mathbf{m}^{*}+\left(-\rho \mathbf{u} \mathbf{u}+\mathbf{B B}+\mathbf{T}_{N}\right) \cdot \mathbf{m}^{*}\right] \cdot d \mathbf{S} .
$$

This surface integral represents the total stress in the direction of $\mathbf{m}^{*}$, normal to the boundary. The integrand of the first term on the righthand side of Eq. (12) reads

$$
-\mathbf{T}: \nabla \mathbf{m}^{*}=\rho \mathbf{u} \cdot\left(\mathbf{u} \cdot \nabla \mathbf{m}^{*}\right)-\mathbf{B} \cdot\left(\mathbf{B} \cdot \nabla \mathbf{m}^{*}\right)+\left(p+\frac{B^{2}}{2}-\lambda \nabla \cdot \mathbf{u}\right) \nabla \cdot \mathbf{m}^{*}-\mathbf{T}_{N}: \nabla \mathbf{m}^{*},
$$

where we used that $\mathbf{g}: \nabla \mathbf{m}^{*}=\nabla \cdot \mathbf{m}^{*}$.

\subsubsection{Induction equation}

Transforming Eq. (1d) to weak form, using

$$
\eta \mathbf{A}^{*} \cdot \nabla \times \mathbf{B}=\nabla \cdot\left(\mathbf{B} \times \eta \mathbf{A}^{*}\right)+\mathbf{B} \cdot \nabla \times \eta \mathbf{A}^{*},
$$

we obtain

$$
\int \mathbf{A}^{*} \cdot \frac{\partial \mathbf{A}}{\partial t} d V=\int\left[\mathbf{A}^{*} \cdot(\mathbf{u}+\nabla \eta) \times \mathbf{B}-\eta \mathbf{B} \cdot \nabla \times \mathbf{A}^{*}\right] d V+\int \eta \mathbf{A}^{*} \times \mathbf{B} \cdot d \mathbf{S} .
$$

\subsection{Boundary conditions}

In this paper we will primarily use the simplest possible boundary conditions. We will fix all the primitive variables at the boundary:

$$
\frac{\partial}{\partial t}(\rho, T, \mathbf{u}, \mathbf{A})=\mathbf{0} .
$$

These boundary conditions are imposed for convenience. They allow internal modes. More general boundary conditions will have to be taken into account when modeling for example the divertor, where plasma can freely flow out of the domain. Such outflow conditions have already been implemented for the reduced MHD models in [26]. Also to simulate a resistive wall mode, different boundary conditions should be used, which requires evaluation of the surface terms of the previous sections. See [27] for an effort to simulate resistive wall modes. The conditions (17) can be shown to describe an ideally conducting wall, impermeable for both plasma and electric current. When no current can penetrate the boundary,

\footnotetext{
1 An alternative weak formulation can be obtained for the hyperdiffusive term. Integrating by parts with respect to the divergence operators acting on $D_{h} \nabla \rho^{*}$, we obtain the alternative weak form

$$
-\int D_{h} \nabla \nabla^{2} \rho \cdot \nabla \rho^{*} d V=\int \nabla \nabla \rho: \nabla D_{h} \nabla \rho^{*} d V-\int D_{h}\left(\nabla \rho^{*} \cdot \nabla \nabla \rho\right) \cdot d \mathbf{S}
$$


Ohm's law (8) with $\mathbf{u}=\mathbf{0}$ shows that the normal component of the electric field vanishes. When the boundary of the domain consists of an ideally conducting wall also the tangential component of the electric field vanishes. With Eq. (7), the boundary condition $\partial \mathbf{A} / \partial t=\mathbf{0}$ then follows.

The unknowns are the changes of the primitive variables from one time step to the next. Therefore the conditions (17) constitute simple Dirichlet boundary conditions. Because the test functions should live in the same space as the unknowns, Dirichlet boundary conditions also hold for the test functions. We can use this property to neglect almost all boundary integrals of the weak formulations derived in the previous section. In the code this is implemented in the matrix equation that results from the discretization, by taking out those lines corresponding to the basis functions that do not satisfy the boundary conditions. These lines are then replaced by conditions that implement the boundary conditions (17).

The only surface term that does not necessarily vanish is the hyperdiffusive term in Eq. (11). By not taking into account this boundary term, the natural boundary condition $\nabla^{2} \rho=0$ is implemented. This is a boundary condition that seems reasonable in the sense that it will probably not alter the interior solution too much.

\subsection{Summary of the weak forms}

When the test functions vanish at the boundary, all the surface integrals in the above weak formulations disappear. The weak formulation can be summarized as

$$
\begin{aligned}
\int \rho^{*} \frac{\partial \rho}{\partial t} d V & =\int\left[\left(\rho \mathbf{u}+\mathbf{q}_{m}\right) \cdot \nabla \rho^{*}+\rho^{*} S_{m}\right] d V, \\
\int \mathbf{m}^{*} \cdot \frac{\partial \rho \mathbf{u}}{\partial t} d V & =\int\left[\rho \mathbf{u} \cdot\left(\mathbf{u} \cdot \nabla \mathbf{m}^{*}\right)-\mathbf{B} \cdot\left(\mathbf{B} \cdot \nabla \mathbf{m}^{*}\right)+\left(p+\frac{1}{2} B^{2}-\lambda \nabla \cdot \mathbf{u}\right) \nabla \cdot \mathbf{m}^{*}-\mathbf{T}_{N}: \nabla \mathbf{m}^{*}\right] d V, \\
\int p^{*} \frac{\partial p}{\partial t} d V & =\int\left[\left[\gamma p \mathbf{u}+(\gamma-1) \mathbf{q}_{h}\right] \cdot \nabla p^{*}+(\gamma-1) p^{*}\left(\mathbf{u} \cdot \nabla p+\eta J^{2}+\mathbf{T}_{v}: \nabla \mathbf{u}+S_{h}\right)\right] d V, \\
\int \mathbf{A}^{*} \cdot \frac{\partial \mathbf{A}}{\partial t} d V & =\int\left[\left(\nabla \times \mathbf{A}^{*}\right) \cdot \eta \mathbf{B}-\mathbf{A}^{*} \cdot(\mathbf{u}+\nabla \eta) \times \mathbf{B}\right] d V,
\end{aligned}
$$

where the hyperdiffusive term in $\mathbf{q}_{m}$ will be partially integrated once more as indicated in Eq. (11).

Alternatively, for comparison, we also implement a more primitive formulation in which only partial integration is used when it reduces the order. The magnetic terms are left unaltered:

$$
\begin{aligned}
\int \rho^{*} \frac{\partial \rho}{\partial t} d V= & \int\left[\rho^{*}\left[-\nabla \cdot(\rho \mathbf{u})+S_{m}\right]+\mathbf{q}_{m} \cdot \nabla \rho^{*}\right] d V \\
\int \mathbf{m}^{*} \cdot \frac{\partial \rho \mathbf{u}}{\partial t} d V= & \int\left[\mathbf{m}^{*} \cdot[-\mathbf{u} \nabla \cdot(\rho \mathbf{u})-\rho \mathbf{u} \cdot \nabla \mathbf{u}-\nabla p]+\left(\frac{1}{2} B^{2}-\lambda \nabla \cdot \mathbf{u}\right) \nabla \cdot \mathbf{m}^{*}\right. \\
& \left.-\mathbf{B} \cdot\left(\mathbf{B} \cdot \nabla \mathbf{m}^{*}\right)-\mathbf{T}_{N}: \nabla \mathbf{m}^{*}\right] d V, \\
\int p^{*} \frac{\partial p}{\partial t} d V= & \int\left[p^{*}\left[-\gamma p \nabla \cdot \mathbf{u}-\mathbf{u} \cdot \nabla p+(\gamma-1)\left(\eta J^{2}+\mathbf{T}_{v}: \nabla \mathbf{u}+S_{h}\right)\right]\right. \\
& \left.+(\gamma-1) \mathbf{q}_{h} \cdot \nabla p^{*}\right] d V, \\
\int \mathbf{A}^{*} \cdot \frac{\partial \mathbf{A}}{\partial t} d V= & \int\left[\nabla \times \mathbf{A}^{*} \cdot \eta \mathbf{B}-\mathbf{A}^{*} \cdot(\mathbf{u}+\nabla \eta) \times \mathbf{B}\right] d V,
\end{aligned}
$$

where we left out the same surface integrals as before. In some test cases, notably the artificial compressibility test case shortly mentioned in Section 5.1 and considered in more detail in [28], this formulation is found to be more stable.

\subsection{Coordinate system}

We use the cylindrical coordinate system for toroidal problems $(R, Z, \phi)$. Being a righthanded coordinate system, this differs from the standard cylindrical coordinate system $(R, \phi, Z)$ in the direction of $\nabla \phi$.

With $\mathbf{a}_{3} \equiv(\partial \mathbf{r} / \partial \phi)=R \hat{\mathbf{e}}_{\phi}$ we have $A_{3} \equiv \mathbf{a}_{3} \cdot \mathbf{A}=R A_{\phi}$. In an axisymmetric equilibrium, $A_{3}$ can be identified with the poloidal magnetic flux function $\psi$, that will be introduced in Eq. (22). Therefore we use this covariant component of $\mathbf{A}$ as a primitive variable. For the velocity however we use the physical component $u_{\phi}=\hat{\mathbf{e}}_{\phi} \cdot \mathbf{u}$. Our primitive variables then read

$$
\mathbf{y}=\left(A_{R}, A_{Z}, A_{3}, u_{R}, u_{Z}, u_{\phi}, \rho, T\right) .
$$

For scalar test functions like $\rho^{*}$ we will use the basis functions (Galerkin method). For the vectorial test functions $\mathbf{m}^{*}$ and $\mathbf{A}^{*}$ we use 


$$
\begin{aligned}
\mathbf{m}^{*} & =u_{R}^{*} \hat{\mathbf{e}}_{R}+u_{Z}^{*} \hat{\mathbf{e}}_{Z}+u_{\phi}^{*} \hat{\mathbf{e}}_{\phi}, \\
\mathbf{A}^{*} & =A_{R}^{*} \hat{\mathbf{e}}_{R}+A_{Z}^{*} \hat{\mathbf{e}}_{Z}+A^{3 *} \mathbf{a}_{3},
\end{aligned}
$$

where for the components $u_{R}^{*}$, $u_{Z}^{*}$, etc., we will also simply use the basis functions. Inserting these expressions into the weak formulations and subsequently choosing only one of the six test functions to be nonzero gives six scalar equations. After this step it is a matter of working out the integrands of the weak formulation explicitly in the $(R, Z, \phi)$-coordinate system to obtain a formulation that is suitable for implementation.

\subsection{Dimensions}

Introducing a characteristic length $l_{0}$, a characteristic magnetic field strength $B_{0}$, a characteristic density $\rho_{0}$, velocities normalized by the Alfvén velocity $v_{A}=B_{0} / \sqrt{\mu_{0} \rho_{0}}$ and pressures normalized by $\rho_{0} v_{0}^{2}=B_{0}^{2} / \mu_{0}$, in the ideal MHD equations $\mu_{0}$ is divided out [29]. Non-ideal terms are non-dimensionalized by dividing the diffusion coefficients by $l_{0} v_{A}$. Often Alfvén units are used in which the tokamak minor radius, toroidal magnetic field and density on the magnetic axis are used as characteristic values. We take $\mu_{0}=1$, but no explicit non-dimensionalization is applied to the input or output.

Note that by defining $T=p / \rho$ we absorb the factor $k_{B} / m$, with $m$ the average particle mass, into our definition of the temperature.

\subsection{Initial conditions}

For an axisymmetric MHD equilibrium, the magnetic field can be written as

$$
\mathbf{B}=\nabla \phi \times \nabla \psi+F(\psi) \nabla \phi,
$$

with $F \equiv R B_{\phi}$. The stationary, axisymmetric, ideal form of the MHD equations (1a)-(1d) with purely toroidal rotation $\mathbf{u}=$ $R \Omega \hat{\mathbf{e}}_{\phi}$ can, using Eq. (22), be reduced to the extended Grad-Shafranov equation

$$
R^{2} \nabla \cdot\left(R^{-2} \nabla \psi\right)=-F \frac{d F}{d \psi}-\left.R^{2} \frac{\partial p}{\partial \psi}\right|_{R}
$$

This equation represents the force balance normal to the magnetic surfaces labeled by $\psi$. Within these magnetic surfaces, the force balance is given by

$$
\left.\frac{\partial p}{\partial R}\right|_{\psi}=\rho R \Omega^{2} .
$$

We assume that $p \rho^{-\gamma_{e}}$ is a function of $\psi$ only. Here, $\gamma_{e}$ is the adiabatic constant of the equilibrium [30]. Eq. (24) can then be solved analytically by

$$
\begin{aligned}
& p=p_{0}(\psi)\left(1+\frac{\rho_{0}\left(R^{2}-R_{0}^{2}\right) \Omega^{2}}{2 p_{0} \zeta_{\gamma}}\right)^{\zeta_{\gamma}}, \\
& \rho=\rho_{0}(\psi)\left(1+\frac{\rho_{0}\left(R^{2}-R_{0}^{2}\right) \Omega^{2}}{2 p_{0} \zeta_{\gamma}}\right)^{\zeta \gamma-1} .
\end{aligned}
$$

Different choices of $\gamma_{e} \equiv \zeta_{\gamma} /\left(\zeta_{\gamma}-1\right)$ lead to different quantities being constant on the flux surfaces. The most natural choice for tokamak applications is $\gamma_{e}=1$ in which case the temperature $T=p_{0} / \rho_{0} \equiv T_{0}$ is constant on the magnetic surfaces.

As input we supply the parametric dependencies of $T_{0}, \rho_{0}, F$, and $\Omega$ on $\psi_{n}$, with $\psi$ normalized between zero and one. The extended Grad-Shafranov equation (23) is then solved with the boundary condition $\psi=0$. We implement the parameterizations of Eqs. (25a) and (25b) for the pressure and the density for a general equilibrium adiabatic constant $\gamma_{e}$. The resulting $\psi(R, Z), T(\psi, R), \rho(\psi, R), F(\psi)$, and $\Omega(\psi)$ are then used as initial conditions for the simulation, in particular $A_{3}=\psi, u_{\phi}=R \Omega$, and

$$
\mathbf{F}=F(\psi) \nabla \phi .
$$

The equilibrium is calculated on a polar grid of $N_{r}$ elements in the radial direction and $N_{\theta}$ elements in the poloidal direction. The grid used for the simulations is typically aligned with the equilibrium magnetic field, obtained by accurate interpolation from the polar grid, and can have a different number of radial and poloidal elements $N_{\psi}$ and $N_{\vartheta}$ respectively. The equilibrium is then recalculated on the new grid to ensure an accurate representation. Note that including also poloidal flow in the equilibrium would require much more effort than required for including only toroidal flow. See for example [31] on how this could be done.

In an ideal equilibrium with toroidal rotation $\mathbf{u}=R \Omega \hat{\mathbf{e}}_{\phi}$ there exists an electric field (8) 


$$
\mathbf{E}=-\mathbf{u} \times \mathbf{B}=\Omega \nabla \psi .
$$

A scalar potential $\Phi(\psi)=\int \Omega(\psi) d \psi$ can be conveniently introduced to describe such an electric field. In the present gauge (7), this equilibrium electric field is described by a magnetic vector potential $\mathbf{A}$ that increases linearly in time. A timevarying quantity is therefore an inherent part of the equilibrium.

A problem with this is that sometimes we want to study for example the linear growth rate of an instability, while keeping fixed the $n=0$ harmonic provided by the initial conditions. If we do this by restraining the primitive variables from changing in time, we effectively remove the equilibrium electric field. Another problem is that when there is plasma flow near the boundary, the normal component of $\mathbf{A}$ has to change in time, in violation of our boundary conditions (17). For these reasons, when including equilibrium rotation, we prefer to add the static equilibrium electric field $\Omega \nabla \psi$ directly as a source to the righthand side of Eq. (1d).

Often a next step is to compute axisymmetrically, using only $n=0$. When the ideal MHD equations are used, the variables should not evolve in this case. This is because the initial conditions are an equilibrium solution of these equations. With the Dirichlet boundary conditions this equilibrium is also stable to $n=0$ perturbations. To verify that this is indeed the case actually provides a good first test of the implementation of the equations.

Often we want to start a simulation from a non-ideal equilibrium with various diffusivities and resistivities. In the corresponding steady state however some of the profiles may have completely diffused away. To avoid this we can introduce sources in the equations that balance the diffusion. In tokamak simulations, the diffusivities are often so low, and the time scales of interest so fast, that for practical purposes the ideal initial conditions provide a quasi-equilibrium.

\section{Numerical method}

We use a mixed spectral / finite element spatial representation. A real sine-cosine Fourier expansion is used in the toroidal direction. Finite elements are used in the poloidal plane [21].

A general multi-step method, allowing for second-order accurate fully implicit time integration, is used. The MHD equations can be quite stiff; the three fundamental waves in MHD for example, can have widely varying frequencies. When we are not interested in the highest of these frequencies, a very attractive feature of so-called L-stable implicit methods [32] is that the time step can be chosen much larger than what is required to resolve the highest frequencies.

The integrals of the weak formulation are calculated per element and put into an element matrix. These element matrices are then combined in an assembly stage after which the Dirichlet boundary conditions (17) are imposed. The resulting sparse system of linear equations is saved in coordinate format and is solved using the direct solver Pastix [33].

In addition, an iterative solver has been implemented based on the Generalized Minimal RESidual (GMRES) method. A preconditioner has been implemented based on the fact that, because of the axisymmetry of the initial conditions, the various Fourier harmonics are initially primarily influenced by the $n=0$ equilibrium component [22]. Because the inversion of the sub-matrices for different harmonics can be done independently, this allows for an easily parallelizable solver.

The integrals of the weak formulation are split into separate contributions from the individual elements. Because the basis functions are nonzero only in the elements bordering the node at which the unknown is defined, this gives a small element matrix and element vector. These are constructed on separate processors for separate groupings of elements.

A fourth-order accurate Galerkin method based on the Bézier formalism is used. This approach has been described in detail in [21]. Here we recollect only those details relevant for the present exposition.

The integrals of the weak formulation are not evaluated in $(R, Z, \phi)$-coordinates, but only after a coordinate transformation to a unit square. An isoparametric formulation is used in which the coordinates are transformed using the same basis functions as those used for the other variables. An arbitrary quantity, coordinate or variable, is expanded on a unit square as

$$
P(s, t)=\sum_{i=1}^{4} \sum_{j=1}^{4} p_{i, j} \sigma_{i, j} b_{i, j}(s, t),
$$

where the coordinates $s$ and $t$ run from 0 to 1 . The outer sum runs over the four vertices of the element, while the inner sum runs over the four basis functions $b_{i, j}(s, t)$ per vertex. The coefficients $p_{i, j}$ are the unknowns living on the vertices, while the quantities $\sigma_{i, j}$ arrange the continuity of $P$ across elements. In the local coordinates $(s, t)$ this continuity is only geometric [21]. In the Cartesian coordinates $(R, Z)$ however, the variables are continuously differentiable or $C^{1}$. The basis functions or shape functions $b_{i, j}(s, t)$ are products of cubic polynomials in $s$ and $t$. They follow from Bernstein polynomials after taking into account continuity requirements at the edges of the elements [21]. For the first vertex $i=1$ :

$$
\begin{aligned}
& b_{1,1}(s, t)=(1-s)^{2}(1-t)^{2}(1+2 s)(1+2 t), \\
& b_{1,2}(s, t)=3(1-s)^{2}(1-t)^{2}(1+2 t) s \\
& b_{1,3}(s, t)=3(1-s)^{2}(1-t)^{2}(1+2 s) t \\
& b_{1,4}(s, t)=9(1-s)^{2}(1-t)^{2} s t
\end{aligned}
$$




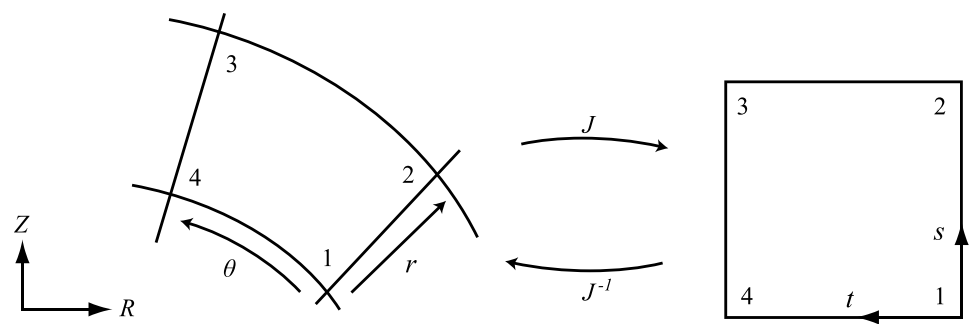

Fig. 1. A schematic representation of the coordinate transformation from an element in $(R, Z)$ to a reference unit square with coordinates ( $s, t)$.

For the second vertex, referring to Fig. 1 for the labeling of the vertices, we have the same basis functions with $s \rightarrow 1-s$. That is $b_{2, j}(s, t)=b_{1, j}(1-s, t)$. For the other two basis functions we have $b_{3, j}(s, t)=b_{1, j}(1-s, 1-t)$ and $b_{4, j}(s, t)=$ $b_{1, j}(s, 1-t)$.

\subsection{Spatial discretization}

The basis functions are zero in elements to which the vertex does not belong. All basis functions labeled $i$ vanish on the two edges opposite to the vertex $i$.

Non-axisymmetric variables acquire an additional dependence on the toroidal angle $\phi$ by multiplication of the parameterization (28) with $\cos \left(n_{k} \phi\right)$ or $\sin \left(n_{k} \phi\right)$. This adds another index to the unknowns $p_{i, j} \rightarrow p_{i, j, k}$ and the basis functions $b_{i, j} \rightarrow b_{i, j, k}$. The toroidal mode numbers $n_{k}=(k-1) / 2$ typically run from $n_{k}=0$ for $k=1$ to $n_{k}=n$ for $k=2 n+1$. However, it is also possible to use only a fraction of the full toroidal angle, so that mode numbers $n_{k}$ that are not a multiple of some integer periodicity are excluded. This allows us to focus on a single mode number $n$, only even $n$, only multiples of 3 , etc.

In the Galerkin method, the test functions are equal to the basis functions. The integrands of the weak formulation (18a)-(18d) will therefore contain Fourier harmonics of at most a toroidal mode number $2 n$ and polynomials of sixth-order in $s$ and $t$. Such integrals can be evaluated exactly using a four-point Gaussian integration method [21]. The toroidal integration can be done using a fast Fourier transform. Alternatively, the integration over the toroidal angle can be done exactly with a Riemann sum using $N=4 n$ points. These points are chosen to be evenly distributed over the full toroidal angle so that $\phi_{k}=2 \pi(k-1) / 2 n$. The volume integral of a quantity $f$ is then given by

$$
\int f d V \approx \sum_{\text {elements }} \frac{1}{N} \sum_{k=1}^{N} \sum_{j=1}^{4} \sum_{i=1}^{4} f\left(s_{i}, t_{j}, \phi_{k}\right) w_{i} w_{j} 2 \pi R \operatorname{det}(J),
$$

with $w_{i}$ the weights. The evaluation points satisfy $0<s_{1}<s_{2}$ and $s_{3}=1-s_{2}$ and $s_{4}=1-s_{1}$. The fact that none of the integration points lies on the boundary of the elements has a clear advantage when the elements tend to a position where the determinant

$$
\operatorname{det}(J)=\left|\begin{array}{cc}
R_{s} & R_{t} \\
Z_{s} & Z_{t}
\end{array}\right|=R_{s} Z_{t}-R_{t} Z_{s}
$$

vanishes. The Jacobian matrix $J$ provides the coordinate transformation from the poloidal plane in $(R, Z)$ to the unit square in $(s, t)$, see Fig. 1 . The inverse provides the back-transformation for, for example, the derivatives:

$$
\left(\begin{array}{c}
\partial_{R} \\
\partial_{Z}
\end{array}\right)=J^{-1}\left(\begin{array}{c}
\partial_{s} \\
\partial_{t}
\end{array}\right)=\frac{1}{\operatorname{det}(J)}\left(\begin{array}{cc}
Z_{t} & -Z_{s} \\
-R_{t} & R_{S}
\end{array}\right)\left(\begin{array}{c}
\partial_{s} \\
\partial_{t}
\end{array}\right) .
$$

For second-order derivatives we apply this operation twice, taking into account derivatives of $\operatorname{det}(J)$.

\subsection{Time integration}

\subsubsection{Method}

We consider the most general two-step time-integration method for solving $\dot{P}=Q$. Following the derivation in [34], but allowing for a variable time step, we obtain to first-order

$$
(1+\zeta r) P^{n+1}-(1+\zeta(1+r)) P^{n}+\zeta P^{n-1}=\Delta t_{+}\left(\theta Q^{n+1}+(1+\phi-\theta) Q^{n}-\phi Q^{n-1}\right),
$$

where $\zeta, \theta$, and $\phi$ can be chosen freely. The parameter $r \equiv \Delta t_{-} / \Delta t_{+}$, where $\Delta t_{+}$is the time step when going from the time level $n$ to $n+1$. This can be different from the time step $\Delta t_{-}$used to go from $n-1$ to $n$. For $r=1$ the Beam-Warming scheme [34] is obtained.

The condition for second-order accuracy reads 


$$
\phi r+\theta-\zeta r(1+r)=\frac{1}{2}
$$

Eq. (33) gives the most general two-step method for variable time step. It includes the well-known $\theta$-schemes for $\zeta=\phi=0$, of which forward Euler $\theta=0$, backward Euler $\theta=1$, and Crank-Nicolson $\theta=1 / 2$ are classical examples.

\subsubsection{Properties}

For the model eigenvalue equation $\dot{P}=Q=\lambda P$ [35], the growth factor $P^{n+1} / P^{n}=P^{n} / P^{n-1} \equiv G$ for $r=1$ reads in the limit of large time steps

$$
\lim _{\Delta t \rightarrow \infty} G=\frac{1+\phi-\theta}{2 \theta} \mp \sqrt{\frac{\phi}{\theta}+\left(\frac{1+\phi-\theta}{2 \theta}\right)^{2}} .
$$

Note that in this limit the growth factor becomes independent of $\zeta$. It vanishes for $\phi=0, \theta=1$, which is the backwarddifferencing second-order accurate scheme (BDF2, or Gear's scheme). In this scheme the solution component corresponding to any large eigenvalue $\lambda$, real or imaginary, is heavily damped, unless the time step is sufficiently small. This scheme is therefore suitable for, for example, marching towards an equilibrium. The scheme can also be useful in stiff problems, such as the simulation of a tokamak plasma. With a suitably chosen time step, the fastest waves will be damped while the slower dynamics can be resolved.

Regarding stability of these numerical schemes, it is necessary that $|G|^{2} \leq 1$ when $\operatorname{Re}(\lambda) \leq 0$, in which case the scheme is A-stable. It can be shown [34] that this holds when

$$
\frac{1}{2}+\phi \leq \theta \text { and }-\frac{1}{2} \leq \zeta \leq \theta+\phi-\frac{1}{2} .
$$

When next to A-stability also $G \rightarrow 0$ when $|\lambda \Delta t| \rightarrow \infty$, as is the case for BDF2, the scheme is L-stable.

For the Crank-Nicolson scheme $\theta=1 / 2, \zeta=\phi=0$, the limit of Eq. (35) gives -1 . This implies that the solution can display numerical oscillations $P^{n+1}=-P^{n}=P^{n-1}$.

For real eigenvalues $\lambda>0$ and $\phi=0$ and $\zeta>0$, a positive quantity can be shown to remain positive when

$$
\Delta t<\frac{1+\zeta}{\lambda \theta} .
$$

This provides rather demanding conditions on the time step for assuring positivity.

\subsubsection{Implementation}

The integrands of the weak formulation (18a)-(18d) or (19a)-(19d) can be written in matrix form as

$$
\frac{\partial \mathbf{P}(\mathbf{y})}{\partial t}=\mathbf{Q}(\mathbf{y}, t),
$$

with each of the entries of this vector equation representing one of the scalar MHD equations.

Nonlinear terms are treated using linearization,

$$
\mathbf{Q}^{n+1}=\mathbf{Q}^{n}+\left(\frac{\partial \mathbf{Q}}{\partial \mathbf{y}}\right)^{n} \cdot \delta \mathbf{y}^{n}+O\left(\Delta t^{2}\right)
$$

where $\delta \mathbf{y}^{n} \equiv \mathbf{y}^{n+1}-\mathbf{y}^{n}$. Similarly for $\mathbf{Q}^{n-1}, \mathbf{P}^{n+1}$ and $\mathbf{P}^{n-1}$. The matrix $\partial \mathbf{Q} / \partial \mathbf{y}$ is the Jacobian that contains the derivatives of all the righthand sides of the equations with respect to all the primitive variables. These derivatives are evaluated analytically in our method.

After linearization, the two-step scheme (33) reads with $\Delta t=\Delta t_{+}$

$$
\left[(1+\zeta r)\left(\frac{\partial \mathbf{P}}{\partial \mathbf{y}}\right)^{n}-\Delta t \theta\left(\frac{\partial \mathbf{Q}}{\partial \mathbf{y}}\right)^{n}\right] \cdot \delta \mathbf{y}^{n}=\Delta t \mathbf{Q}^{n}+\left(\zeta\left(\frac{\partial \mathbf{P}}{\partial \mathbf{y}}\right)^{n}+\phi \Delta t\left(\frac{\partial \mathbf{Q}}{\partial \mathbf{y}}\right)^{n}\right) \cdot \delta \mathbf{y}^{n-1} .
$$

This matrix equation is solved for the unknowns $\delta \mathbf{y}^{n}$. In the remainder of this work we will use $\phi=0$.

3.2.4. Test

In Fig. 2 we show the error after numerically integrating

$$
\frac{d \rho(t)}{d t}=\rho(t) \quad \text { with } \quad \rho(0)=1,
$$

from $t=0$ up to $t=1$.

Using a single time step, the implicit Euler scheme diverges. This can be understood from Eq. (40), because with $\partial P / \partial y=$ $\partial Q / \partial y=\partial \rho / \partial \rho=1, \theta=1, \zeta=\phi=0$, and $\Delta t=1$, the term between square brackets vanishes, while the righthand side is finite. 


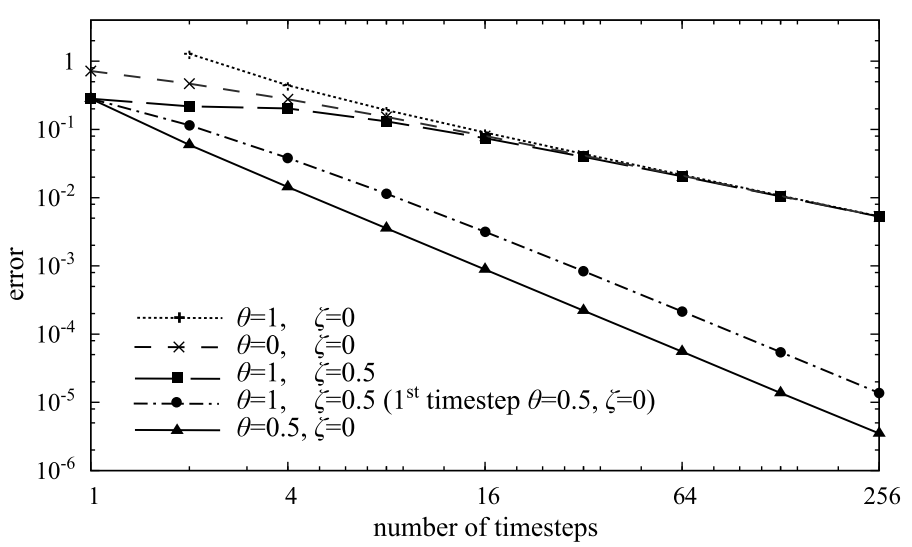

Fig. 2. The error $|\rho(1)-e|$, when calculating $d \rho(t) / d t=\rho$ with $\rho(0)=1$, using various time-integration schemes. The first two methods give first-order convergence, while the final Crank-Nicolson method shows second-order convergence. The BDF2 scheme with $\theta=1$ and $\zeta=0.5$ only shows second-order convergence when for the first time step a different method with $\zeta=0$ is used.

When $\zeta$ is nonzero, the time-integration scheme (33) requires $P^{n-1}$, or equivalently Eq. (40) requires $(\partial \mathbf{P} / \partial \mathbf{y})^{n-1}$. At the start of a simulation this information of the previous time level is not available, which endangers the properties of the scheme. This is the reason why the second-order BDF2 scheme $(\theta=1, \zeta=1 / 2)$ only shows first-order convergence in Fig. 2. It is well-known that the accuracy of the approximation for $P^{n-1}$ can be at most an order lower in the time step than that required for the remainder of the time evolution. For example, $P^{n-1}$ should be supplied with an accuracy of at least first-order in time to ensure second-order accuracy for the remainder of the time evolution. To supply such an approximation we can use any first-order scheme. Using Crank-Nicolson for the first time step recovers the expected second-order convergence.

We note that the same problem occurs when adjusting the time step during the simulation. Using a scheme that has $\zeta=$ 0 for at least one new time step before proceeding with a scheme that has $\zeta$ nonzero ensures the convergence properties of the scheme also in this case.

With $\lambda=1$, Eq. (37) predicts oscillatory behavior for $\Delta t>2$ with Crank-Nicolson and for $\Delta t>1.5$ with BDF2, which was verified to be the case.

\section{Parallel projection}

MHD instabilities in tokamak plasmas avoid to a large degree compression of the toroidal magnetic field because of the large amount of energy this requires. It can require some special care to accurately represent such perturbations numerically. This problem of 'spectral pollution' is the primary reason why for example in the method from [6] the choice is made for a representation of the velocity in terms of a stream function that does not compress the magnetic field.

Our simulations of linear MHD instabilities are found to be polluted by spurious fluctuations in the magnetic vector potential components $A_{R}$ and $A_{Z}$ making up the toroidal magnetic field. This small noise primarily influences the solution through the Lorentz force in the toroidal component of the momentum equation. Before we discuss our internal kink mode simulations in more detail in Section 6.1, in Fig. 3(a) we already show results for this test case. In particular the toroidal velocity can be seen to be dominated by non-physical noise.

This can be avoided by projecting the momentum equation in the direction of the magnetic field, instead of in the toroidal direction. Under such a parallel projection the Lorentz force vanishes. The resulting solutions are much smoother, as is shown in Fig. 3(b).

With the test function

$$
\mathbf{m}_{B}^{*}=\bar{m}_{B}^{*} \mathbf{B}=\bar{m}_{B}^{*}\left(B_{R} \hat{\mathbf{e}}_{R}+B_{Z} \hat{\mathbf{e}}_{R}+B_{\phi} \hat{\mathbf{e}}_{\phi}\right),
$$

we obtain a scalar equation that contains terms from projections in all three directions:

$$
\int \mathbf{m}_{B}^{*} \cdot \nabla(\nabla \cdot \mathbf{u}) d V=\int\left(\nabla \cdot \mathbf{m}_{B}^{*}\right)(\nabla \cdot \mathbf{u}) d V+\int(\nabla \cdot \mathbf{u}) \mathbf{m}_{B}^{*} \cdot d \mathbf{S} .
$$

Since $\nabla \cdot \mathbf{B}=0$ we have that $\nabla \cdot \mathbf{m}_{B}^{*}=\mathbf{B} \cdot \nabla m_{B}^{*}$, which does not require the calculation of derivatives of the magnetic field. Note that of the six test function components of Eqs. (21a) and (21b) we only replace $u_{\phi}^{*} \hat{\mathbf{e}}_{\phi}$. Furthermore we leave our primitive variables the same as in Eq. (20). We thus do not change our basis vectors for the primitive variables, as would be the case in a strict Galerkin approach. It may seem strange that in the projected equations both the primitive variables and their derivatives, through $\mathbf{B}$, appear. As long as $\mathbf{B}$ is however not linearly dependent on the other two projection directions $\hat{\mathbf{e}}_{R}$ and $\hat{\mathbf{e}}_{Z}$, such a choice of projection is allowed and indeed found to be very useful in this case.

There may be some concern about whether the use of implicit time integration for an equation like 

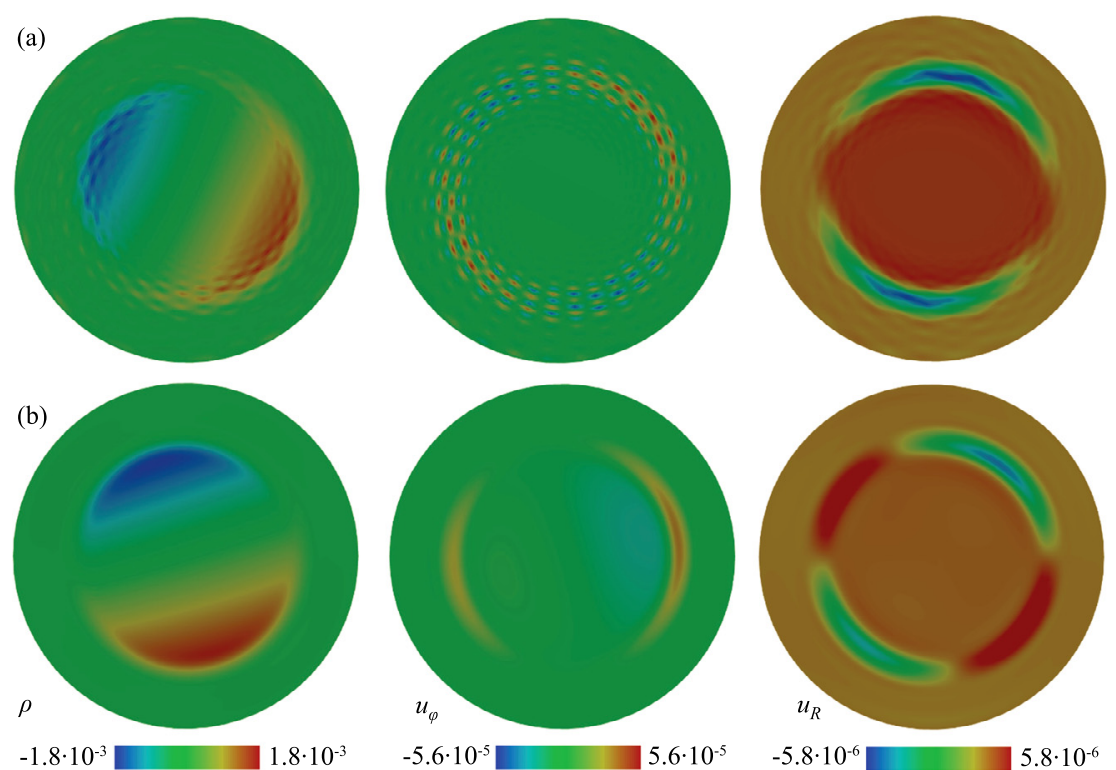

Fig. 3. The results for an internal kink mode simulation with $\eta=10^{-5}$. The numerical artifacts in the variables are caused by weak noise in the Lorentz force. This was found to be avoided when instead of a projection of the momentum equation in (a) the toroidal direction, a projection in (b) the direction of the magnetic field is used.

$$
\mathbf{B} \cdot \frac{\partial \rho \mathbf{u}}{\partial t}=\mathbf{B} \cdot \mathbf{f}
$$

requires special care, because the projection operator can change during a time step. Following the derivation leading up to the time-integration scheme (40) we find that with $\partial \rho u_{R} / \partial t=f_{R}$ and $\partial \rho u_{Z} / \partial t=f_{Z}$ solved, what remains of Eq. (44) is $B_{\phi} \partial \rho u_{\phi} / \partial t=B_{\phi} f_{\phi}$. With $\partial \rho u_{\phi} / \partial t=f_{\phi}$ and $\partial^{2} \rho u_{\phi} / \partial t^{2}=\dot{f}_{\phi}$ to balance the different orders in the time step, the derivation proceeds unaltered, leading to the same time-integration scheme. To show that indeed the second-order time behavior of the parallel projected momentum equation can be guaranteed, in [28], an appropriate test case is considered in detail.

Note that it will be different if we want to solve for example

$$
\rho \frac{\partial \mathbf{u}}{\partial t}=\mathbf{f}
$$

since $\rho$ now appears only on the lefthand side. To second-order in the time step the derivation requires the time derivative of both sides, where $\dot{\rho}$ enters.

\section{Testing individual equations}

\subsection{Vector equations}

Aspects of our implementation of the momentum equation are tested using the analytical solution from [36]. In this paper an analytical solution is derived for the incompressible flow of a Newtonian fluid in a toroidal geometry of large aspect ratio. A toroidal momentum source introduces toroidal rotation and secondary flows in the poloidal plane. For brevity, here we only sketch the outline of the followed approach, which may also be interesting for testing other hydrodynamic and magnetohydrodynamic codes. For details, we refer to [28].

We use a very large aspect ratio, $\epsilon^{-1}=10^{6}$. To ensure incompressibility numerically, we tested both a penalty method by choosing a large value for $\lambda$ and an artificial compressibility method by choosing a large value for $\gamma$. The same analytical solution can also be used to test parts of the induction equation (1d). With $\mathbf{u}=\mathbf{0}$ Eq. (1d) becomes a pure diffusion equation for the magnetic field $\mathbf{B}$ and because $\nabla \cdot \mathbf{B}=0$ the same analytical expression is also a solution of this equation.

Fourth-order convergence of the error in the size of the elements is observed in all cases. We note that the error in the numerical solution primarily originates from the edge. This is because the polynomial basis functions cannot describe circular boundaries exactly [21]. Therefore, the essential boundary condition $\mathbf{u}=\mathbf{0}$ is not exactly prescribed on a circle but on a shape that converges to a circle with an error of order $1 / N_{\theta}^{4}$.

Another notable observation from the artificial compressibility test is that the weak formulation of Eq. (19c), without partial integration of the first-order terms, turns out to be more stable for very high values of $\gamma$ than that of Eq. (18c). Therefore in the following we will use the formulation of Eqs. (19a)-(19d). 


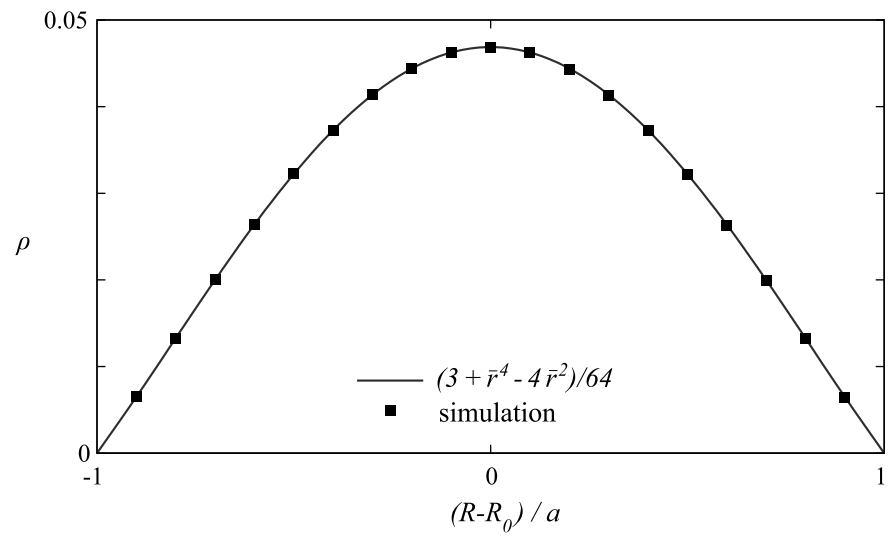

Fig. 4. The profile resulting from solving the stationary hyperdiffusive equation $\nabla^{4} \rho=1$ on a mesh with $N_{r}=N_{\theta}=10$ elements, compared with the analytical solution.

\subsection{Scalar equations}

\subsubsection{Hyperdiffusivity}

To test some aspects of our implementation of the hyperdiffusive term in the continuity equation we investigate a circular tokamak in the cylindrical limit. We look for an analytical solution to the stationary inhomogeneous hyperdiffusion equation $0=\nabla^{4} \rho+S_{\rho}$ for constant $S_{\rho}$. In polar coordinates $(r, \theta)$, assuming symmetry in the $\theta$ and $\phi$ direction, this equation reads

$$
\frac{1}{r} \frac{d}{d r}\left(r \frac{d}{d r}\left(\frac{1}{r} \frac{d}{d r}\left(r \frac{d \rho(r)}{d r}\right)\right)\right)=-S_{\rho} .
$$

The solution that is regular for $r \rightarrow 0$ is given by

$$
\rho(r)=\frac{1}{64}\left(\bar{r}^{2}-\bar{r}^{4}\right) a^{4} S_{\rho}+\rho_{0}\left(1-\bar{r}^{2}\right)+\rho_{a} \bar{r}^{2},
$$

where $\bar{r} \equiv r / a$. With $\rho_{a}=\rho(r=a)=0$ we see that

$$
\left.\nabla^{2} \rho\right|_{\bar{r}=1}=\frac{1}{r} \frac{d}{d r}\left(r \frac{d \rho(r)}{d r}\right)=-4\left(\rho_{0}+\frac{3}{64} S_{\rho}\right) .
$$

When the natural boundary condition is such that $\nabla^{2} \rho$ vanishes at $\bar{r}=1$ the central density becomes $\rho_{0}=-3 S_{\rho} / 64$. Taking a constant density source $S_{\rho}=-1$ and a large aspect ratio $\epsilon^{-1}=100$, the resulting density distribution along a line $Z=0$ is plotted in Fig. 4. The central value can indeed be seen to approach $\rho_{0}=3 / 64 \approx 0.047$. The overall agreement with the analytical result is also good, even on the relatively coarse grid with $N_{r}=N_{\theta}=10$ elements.

\subsubsection{Anisotropic diffusion}

In a fusion plasma the diffusivities in the direction parallel to the magnetic field may easily be many orders of magnitude larger than those perpendicular to the magnetic surfaces. Anisotropies of $O\left(10^{9}\right)$ are not uncommon. A potential problem in numerically simulating such conditions is that a small error in the calculation of the parallel diffusion 'pollutes' the much smaller perpendicular diffusion. Since one of the most valued properties of a fusion device is its ability to keep heat and matter locked up, for some purposes it is important to make sure this can also be made to hold numerically.

A good two-dimensional stationary test case was devised in [1], based on the anisotropic heat diffusion equation

$$
\frac{\partial T}{\partial t}=\nabla \cdot\left[\left(\kappa_{\|}-\kappa_{\perp}\right) \nabla_{\|} T+\kappa_{\perp} \nabla T\right]+S_{T}
$$

The temperature $T$ and poloidal magnetic flux $\psi$ were chosen to have the same analytical dependence on a unit square $-\frac{1}{2} \leq x \leq \frac{1}{2},-\frac{1}{2} \leq y \leq \frac{1}{2}$ :

$$
T, \psi \sim \cos (\pi x) \cos (\pi y) .
$$

Since the magnetic field $\mathbf{B}=\nabla \phi \times \nabla \psi$ is oriented parallel to the iso-contours of the temperature, the parallel diffusion should vanish exactly. When increasing the parallel diffusion coefficient, any deviation from the isotropic case can then be attributed to numerical pollution. Performing this test case, we find no dependence of the error on the amount of parallel diffusion for anisotropies of $O\left(10^{9}\right)$. The fourth-order convergence is completely unaffected by the parallel diffusion coefficient, as it should of course. 


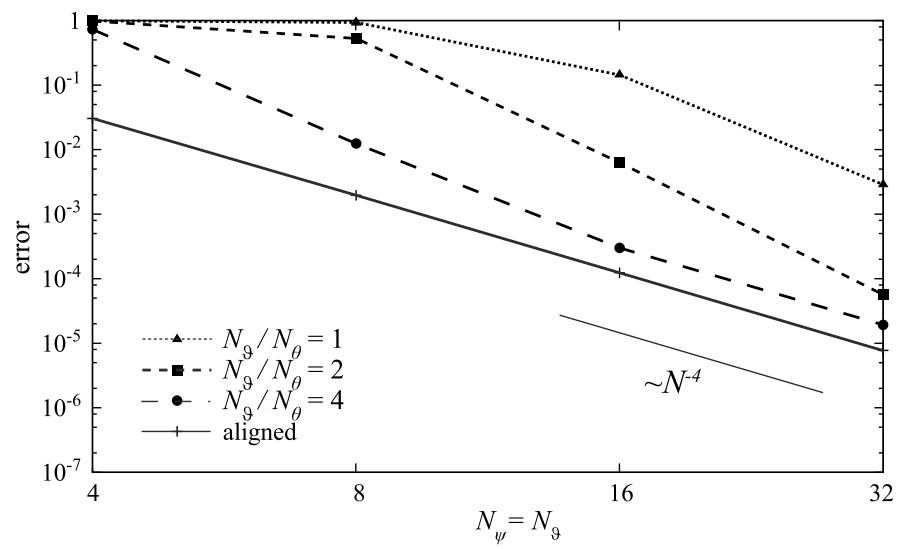

Fig. 5. The maximum difference between the numerical and analytical solution for an anisotropy of $\kappa_{\|} / \kappa_{\perp}=10^{9}$ as a function of the number of elements $N_{\psi}=N_{\vartheta}$, interpolated from a mesh with a potentially different number of poloidal elements $N_{\theta}$. For more information on the difference between $N_{\theta}$ and $N_{\vartheta}$, see section 2.8 .

Interestingly, we do find that the error does increase dramatically with increasing anisotropy when we enter the analytical expressions for $B_{x}=\partial \psi / \partial y, B_{y}=-\partial \psi / \partial x$ directly, instead of obtaining these as numerical derivatives from the analytical expression for $\psi$. In this case we lose four orders of accuracy for an anisotropy of $10^{9}$ compared to the error for the isotropic case. The reason for the fact that this pollution does not occur when we use $\psi$, must lie in the fact that the polluting term,

$$
\mathbf{B} \cdot \nabla T=B_{x} \frac{\partial T}{\partial x}+B_{y} \frac{\partial T}{\partial y}=\frac{\partial \psi}{\partial y} \frac{\partial T}{\partial x}-\frac{\partial \psi}{\partial x} \frac{\partial T}{\partial y},
$$

can be represented very accurately in this case. We would like to test the accuracy with which anisotropic diffusion can be calculated on other grids as well. Therefore we devise a very similar test case as the one that was used on a rectangular domain.

For a constant source $S_{T}$, the isotropic diffusion equation (49) with $\kappa_{\perp}=\kappa_{\|}$and boundary condition $T=0$ is solved on a circular domain by $T(\bar{r})=\left(S_{T} / 4\right)\left(1-\bar{r}^{2}\right)$. With $\psi$ also proportional to $1-\bar{r}^{2}$, the solution of the anisotropic heat equation is again independent of the parallel conductivity $\kappa_{\|}$. We run the diffusion equation to steady state for a single very large time step, with $\theta=1$. It is found again that the error is not influenced by the anisotropy.

The polar grid that is used in this test case is however aligned with the magnetic field. Since anisotropy usually causes problems when the grid is not aligned, we test this as well. To create a non-aligned grid we make use of the machinery that is normally used to create a flux-aligned grid. We set up a toroidal equilibrium with $\epsilon^{-1}=3$ and a Shafranov shift of approximately $0.15 a$ to which we align our grid. This is done using an interpolation from the polar grid on which the equilibrium is calculated. Now we re-run the anisotropic diffusion equation to steady state on this grid that is misaligned with the isocontours of our analytical $\psi$. Now the error does increase with the anisotropy. Fig. 5 compares the case where the grid is aligned with the magnetic field with the case where the grid and magnetic field are not aligned, using an anisotropy of $\kappa_{\|} / \kappa_{\perp}=10^{9}$. The figure also shows what happens when we poloidally refine the initial polar grid from which the misaligned grid is obtained by interpolation. What is found is that when a poloidally much finer mesh is used for this initial polar mesh, the error can be greatly reduced. For an initial mesh that is four times finer in the poloidal direction, the solution approaches that on the aligned mesh, which is again the same as the solution for isotropic diffusion.

This leads us to the conclusion that accurately capturing highly anisotropic diffusion is not a problem when the mesh that is used is of sufficient quality. It does not have to be aligned with the magnetic field, but it has to be set up either from an analytical representation or an accurate numerical interpolation.

\section{Integrated test cases}

The various tests performed in the previous sections were necessary to provide confidence that the equations are implemented correctly and can be solved accurately. Next, we deploy the newly implemented full MHD equations to show that they can be used to simulate various well-known MHD instabilities. For the test cases in this chapter we have used the Crank-Nicolson time integration scheme, Eq. (40) with $\theta=1 / 2, \zeta=\phi=0$.

\subsection{Resistive $m=n=1$ internal kink mode}

We start from an ideal equilibrium with circular cross-section and $\epsilon=0.1$ with the profiles

$$
\rho / \rho_{0}=1-0.9 \psi_{n},
$$



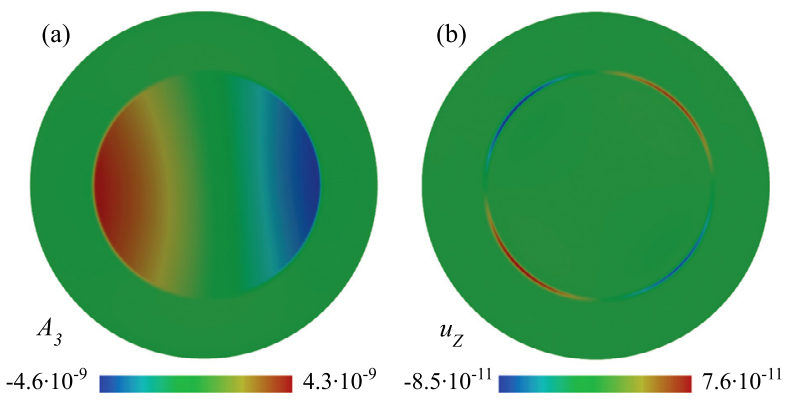

Fig. 6. The perturbed poloidal flux $\psi$ and vertical velocity $u_{Z}$ of an internal kink mode after 50 time steps of $\Delta t=400$ with Crank-Nicolson. Shown is a poloidal cut of the Fourier harmonic $\sim \cos \phi$ at $\phi=0$.

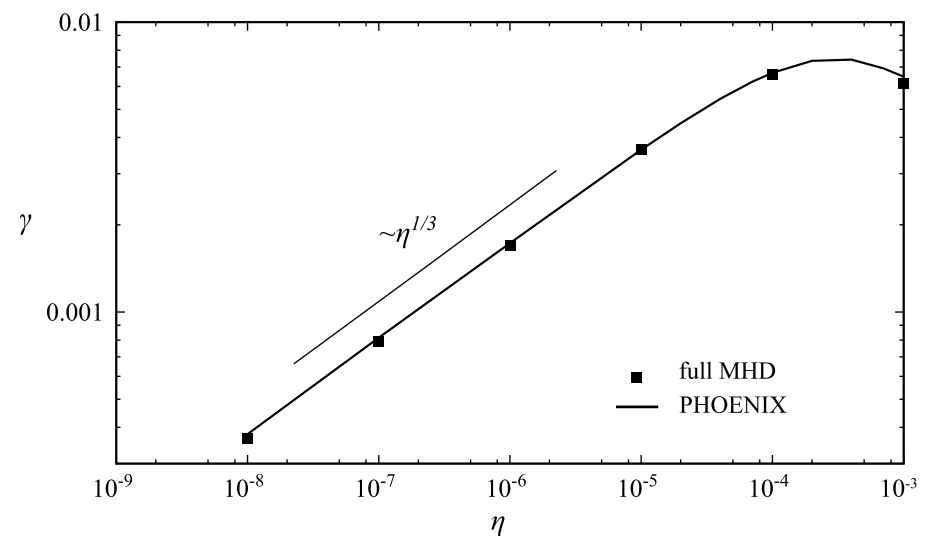

Fig. 7. A comparison of the internal kink mode growth rate as a function of resistivity between the current full MHD method and the linear MHD method coded in PHOENIX.

$$
\begin{aligned}
T / T_{0} & =1-0.8 \psi_{n}, \\
F^{2} & =F_{0}^{2}-4\left(\psi_{e}-\psi_{m}\right)\left(\psi_{n}-0.5 \psi_{n}^{2}\right),
\end{aligned}
$$

where the normalized $\psi_{n} \equiv\left(\psi-\psi_{m}\right) /\left(\psi_{e}-\psi_{m}\right)$ is scaled to zero at the magnetic axis, where $\psi=\psi_{m}$, and unity at the plasma edge, and where $\psi=\psi_{e}$. We use $\rho_{0}=1, T_{0}=2 \cdot 10^{-3}, F_{0}=R_{0}=10$ so that $B_{0}=1$ and $\beta \sim 4 \cdot 10^{-3}$. In this case the units are essentially Alfvén units. The enclosed poloidal flux $\psi_{e}-\psi_{m} \approx 0.48$ is an integral part of the solution to the Grad-Shafranov equation. The safety factor monotonically increases from 0.73 at the axis to 1.6 at the plasma edge.

We use the resistive MHD equations without any additional diffusivities. Under these conditions, the $q=1$ surface becomes unstable against an $n=m=1$ internal kink mode. Kink modes perturb the plasma in a shift-tilt fashion that displaces rather than bends the field lines. In the absence of resistivity the source of their instability is a combination of current and pressure gradient. Because of the relative unimportance of pressure, as indicated by the low $\beta$, we expect for the present test case the current density to be the main source of instability. Resistivity can greatly enhance the growth rate. We fix the equilibrium $n=0$ component and take into account only a single toroidal Fourier harmonic $n=1$. We use an aligned grid of approximately $N_{\psi}=30$ radial and $N_{\vartheta}=30$ poloidal elements, which is refined when necessary to obtain a grid-independent solution. As can be seen from Fig. 6 the structures in the solution can become very fine. Therefore we use local static grid accumulation. Fig. 6 is obtained for $\eta=10^{-8}$ on a grid of $N_{\psi}=N_{\vartheta}=30$ elements, using Gaussian grid accumulation with a width of $0.03 a$.

The structures become finer with smaller resistivity. Both the unstable layer size and the growth rate are known to scale as $\eta^{1 / 3}$. We start from an initial perturbation in the $n=1$ harmonic of the toroidal velocity given by

$$
u_{\phi}^{n=1}=10^{-12} \psi_{n}\left(1-\psi_{n}\right) .
$$

Next we let the equilibrium develop in time, keeping the axisymmetric $n=0$ component fixed. After some time all solution components $y$ show exponential growth with the same growth rate $\gamma=(\partial y / \partial t) / y$. This growth rate is shown in Fig. 7 for various resistivities. Also shown is the growth rate as calculated from the linear MHD code PHOENIX [37,38]. This code uses Fourier harmonics in the poloidal direction. The inclusion of only five poloidal mode numbers is found to be sufficient for accurate results. The equilibrium is calculated with the ideal MHD equilibrium code FINESSE [31], using the same parameters as in Eqs. (52a)-(52c). The current full MHD results and the linear MHD results show good agreement for the full range of resistivities tested. For $\eta<10^{-4}$ the growth rate nicely follows the $\eta^{1 / 3}$ scaling. For higher values of the resistivity, 


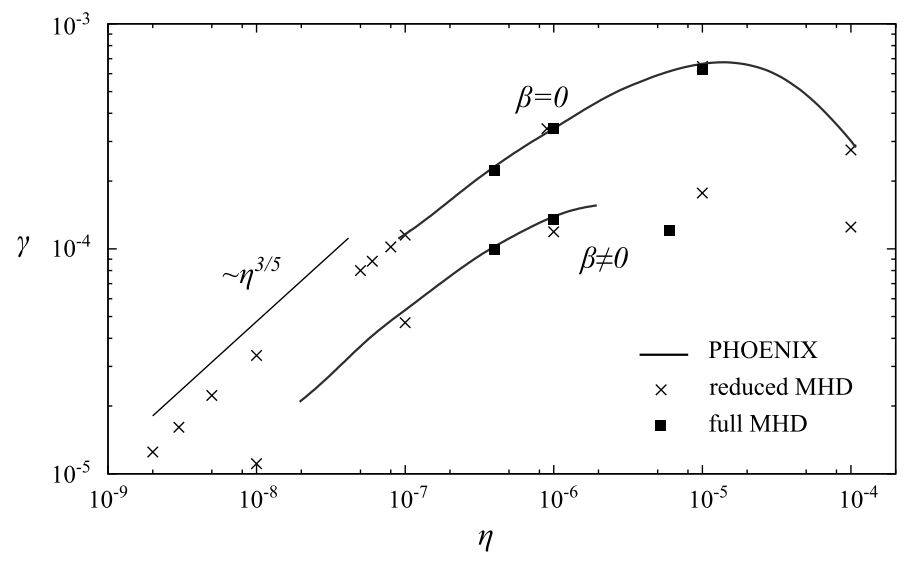

Fig. 8. The growth rate of an $n=1$ tearing mode as a function of the resistivity. Results for the current full MHD method are compared to results for PHOENIX and a reduced MHD method from JOREK. For the $\beta=0$ and $\beta \neq 0$ results a reduced MHD model without and with a toroidal velocity are used respectively.

$\eta>10^{-4}$, the mode width becomes comparable to the machine size and the scaling is lost entirely. This is therefore a good test case of the ideal wall boundary conditions that are also present in PHOENIX. Instead of driving the resistive kink mode, resistivity starts to damp the mode. These resistivities are much too high to be relevant for most tokamak plasmas.

\subsection{Tearing modes}

A next test case is the simulation of tearing modes. These are resistive, current-driven modes, that can become unstable around a rational surface. Tearing modes are primarily driven by the radial variation in the toroidal current density and are typically stabilized by pressure. The growth of these modes is much slower than that of the internal kink mode. Since their growth relies on the 'tearing' of field lines which is only possible for a finite resistivity, the time scale associated with their growth is closer to the resistive time scale than to the Alfvén time scale. For small resistivities this results in a scaling of the growth rate proportional to $\eta^{3 / 5}$.

\subsubsection{Linear simulations}

Zero pressure We start from the same equilibrium as was used for the internal kink simulations of Section 6.1, only for now with zero temperature. We also modify the toroidal magnetic field function to

$$
F^{2}=F_{0}^{2}-4\left(\psi_{e}-\psi_{m}\right)\left(\psi_{n}-0.35 \psi_{n}^{2}\right) .
$$

With $F_{0}=18$ and $\psi_{e}-\psi_{m} \approx 0.4$, this results in a safety factor $q$ between 1.8 and 2.9. The $q=2$ surface is located at $\psi_{n} \approx 0.28$. The $q=3$ surface was intentionally positioned outside the plasma to avoid the occurrence of a second tearing mode. First, we only take into account the evolution of a single $n=1$ harmonic, fixing the axisymmetric $n=0$ component. We start from the same initial perturbation as in Eq. (53), but now for the $n=1$ component of $A_{3}$. The required radial resolution for accurate tearing mode calculations was found to be substantially higher than for the internal kink mode. We restrict ourselves to the calculation of the growth rate for three resistivities, just to demonstrate agreement with the result obtained by PHOENIX, as shown in Fig. 8. Both the resistivity and the growth rate are multiplied by the characteristic magnetic field strength $B_{0}=F_{0} / R_{0}=1.8$ to convert the standard dimensionless Alfvén units used in PHOENIX to the units of the current full MHD method.

Fig. 8 also shows a comparison with a reduced MHD method. This reduced method uses a single stream function to describe the velocity perturbation in the poloidal plane. The method does not allow a velocity component in the toroidal direction. See for example [14] for the specific set of equations that are solved. The figure shows good agreement with PHOENIX's linear MHD results.

For the full MHD model we use a mesh of $N_{\psi}=104, N_{\vartheta}=36$ elements, for which the growth rates are approximately converged to their grid-independent values. We use Gaussian grid refinement with a width depending on the resistivity. It seems to be a general characteristic of the current full MHD method that convergence is achieved from the stable side. That is, the growth rate converges to its grid-independent value from below. This is usually considered to be a favorable property.

The reduced MHD simulations are performed on a mesh with $N_{\psi} \geq 101, N_{\vartheta} \geq 41$; at this resolution the growth rate has converged. Note that there is no particular reason why a slightly different number of elements were used compared to the full MHD simulations.

As the resistivity increases beyond some critical value, damping becomes more important than driving and the growth rate decreases. At the same time, the eigenvalue obtained by PHOENIX acquires an imaginary component, representing 

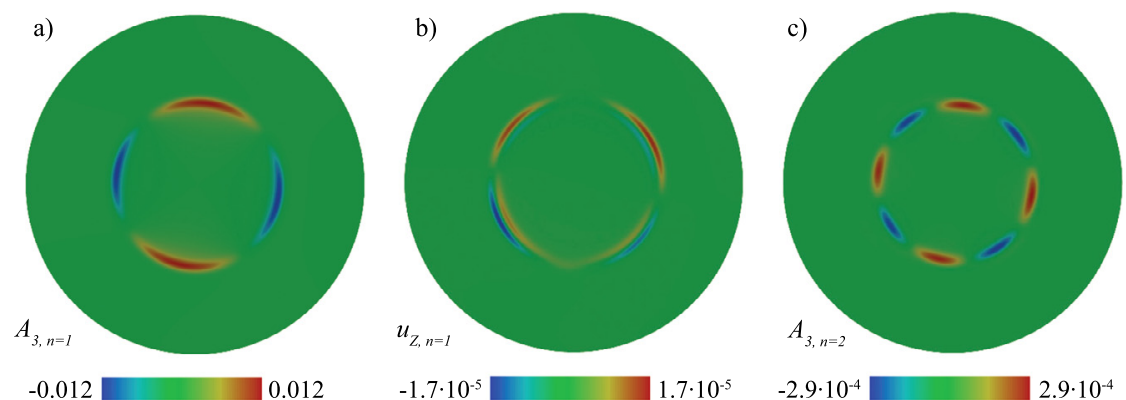

Fig. 9. The $\cos \phi$ component of (a) the perturbed $A_{3}$, (b) $u_{Z}$, and (c) the $\cos 2 \phi$ component of the perturbed $A_{3}$ of a tearing mode in the finite pressure equilibrium.

oscillatory behavior. These oscillations are indeed also observed in the full MHD simulations. The growth rates shown for the higher resistivities represent an average over several of these oscillations.

Outside of a reasonable middle-range of tokamak resistivities, the linear MHD code PHOENIX exhibits difficulty to converge to an eigenvalue with a sufficiently small residual. For this reason the PHOENIX results in Fig. 8 are not shown over the full broad range of resistivities where full MHD results are calculated.

Nonzero pressure Next we introduce a small equilibrium pressure. When the safety factor is above one, the average magnetic field curvature becomes favorable, causing a finite pressure to exert a stabilizing influence. We design a pressure profile that is small enough to allow the instability to grow, but large enough to have a significant influence on the tearing mode growth rate. We choose the same temperature profile as in Eq. (52b). With $T_{0}=4.3 \cdot 10^{-4}$ and $B_{0}=F_{0} / R_{0}=19.5 / 10$ this results in a value of $\beta$ on the magnetic axis of $\beta_{m}=2 p_{m} / B_{0}^{2}=2.3 \cdot 10^{-4}$. Using the same parameters in an equilibrium constructed with FINESSE, allows again a comparison with the growth rates calculated by the linear MHD code PHOENIX.

Comparing the zero- and finite- $\beta$ curves in Fig. 8 we see that indeed the growth rates are lowered by the finite pressure. The growth rates obtained with the current full MHD method for three different resistivities are again found to converge to the linear MHD results.

Finite- $\beta$ growth rates calculated with the simplest reduced MHD model available to us, that was successfully used to obtain the zero- $\beta$ tearing mode growth rates, are almost an order of magnitude smaller than those of the full MHD model. A second reduced MHD model, that includes a toroidal velocity component, produces results that agree reasonably well with the linear and the full MHD results. These are the finite- $\beta$ results plotted in Fig. 8. The growth rates obtained with this reduced MHD model are still slightly lower than those obtained with the full MHD model. Special care has been taken to ensure convergence in the number of elements and the time step. Therefore this small deviation may be attributed to a genuine difference between full and reduced MHD models.

Figs. 9(a)-(b) show the $n=1$ mode structure, which has a clear dominant $m=2$ structure that can be compared with the internal kink mode structure in Fig. 6.

\subsubsection{Nonlinear simulations}

$n=2$ During the linear growth phase of the tearing mode, we add another toroidal Fourier harmonic $n=2$. This harmonic is not unstable by itself, but arises due to nonlinear coupling with the $n=1$ harmonic. After some time, the magnitude of the physical variables of the $n=2$ harmonic are found to be approximately given by the square of those of the dominant $n=1$ harmonic. Correspondingly the growth rate is found to be twice as large as that of the $n=1$ harmonic. Fig. 9(c) clearly shows the $m=4$ structure that is expected for a mode that is resonant with $q=m / n=4 / 2$.

$n=0$ When we follow the evolution of a tearing mode into the nonlinear phase, we would like to take into account the evolution of the axisymmetric component as well. Using $\eta=10^{-6}$, the force equilibrium quickly changes. As the current resistively diffuses, the safety factor goes up and the $q=3$ surface enters the plasma. This allows an associated $3 / 1$ tearing mode to grow. To counteract the resistive diffusion of the $n=0$ component we add a current source. This is realized by changing the resistive term in the induction equation from $\eta \mathbf{J}$ to $\eta\left(\mathbf{J}-\mathbf{J}_{e q}\right)$, with $\mathbf{J}_{e q}$ the equilibrium current density. This change allows the ideal equilibrium solution to be an equilibrium solution of the resistive static equations as well.

$n=1 \quad$ By perturbing the magnetic field, the instability changes the topology of the previously nested magnetic surfaces. By tracing the magnetic field lines around the torus for many times, new magnetic surfaces become visible. The resulting Poincaré plot is shown in Fig. 10. The field lines form new nested surfaces inside magnetic islands.

In reduced MHD the magnetic field is described by Eq. (2) with $F$ constant in space and time. We consider a single helical perturbation $f\left(\psi_{0}, m \vartheta-n \phi\right)$ with $\psi_{0}$ the equilibrium value of $\psi$ and the coordinate $\vartheta$ such that the equilibrium field lines satisfy $d \phi / d \vartheta=q\left(\psi_{0}\right)$. Then we can show that $\mathbf{B} \cdot \nabla \psi^{*}=0$ with the helical flux

$$
\psi^{*}=\psi-\frac{n}{m} \int q\left(\psi_{0}\right) d \psi_{0}
$$




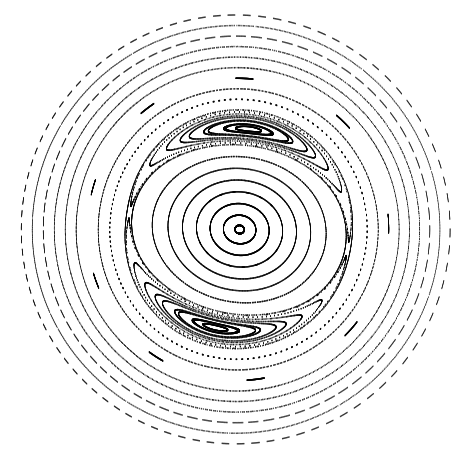

Fig. 10. A Poincaré plot of the magnetic island in the saturation phase of Fig. 12. The starting values are homogeneously distributed radially, but with a higher density near the island. The magnetic field lines are followed for only 1000 turns, explaining why at certain radii close to rational magnetic surfaces the magnetic surfaces are not yet fully traced out. For example the seventh circle from the outside shows nine dashes, indicative of its closeness to a value of $q$ where the field lines go around toroidally nine times for an integer number of poloidal turns.

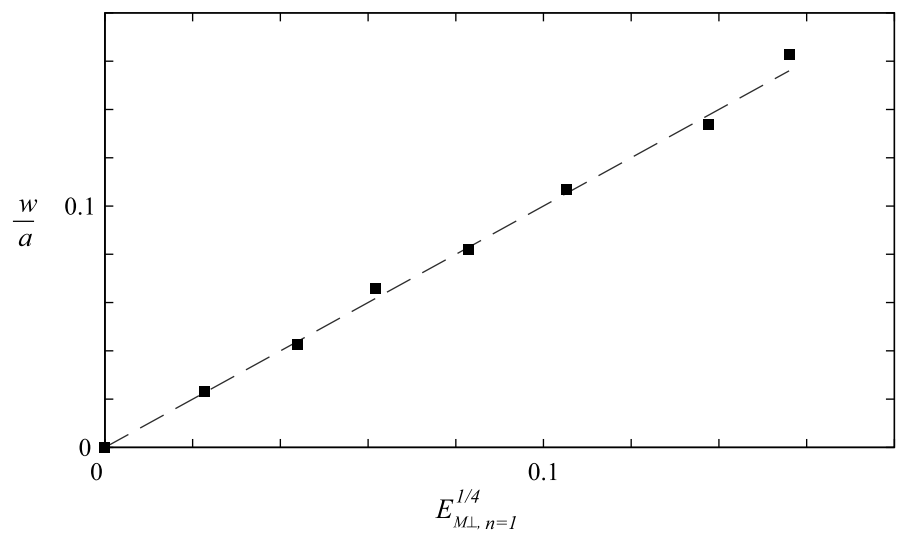

Fig. 11. The normalized island width $w / a$ as estimated from Poincare plots compared to the quartic root of $E_{M \perp, n=1}$, the normalized magnetic energy in the $n=1$ Fourier harmonic of the poloidal magnetic field.

For tearing modes this quantity can be nicely used to visualize the magnetic islands. In full MHD however this is no longer true. It turns out that at a given time in the tearing mode simulations the perturbed $A_{3}$ of the full MHD model is much larger than the perturbed $\psi$ in the reduced MHD models. The other components $A_{R}$ and $A_{Z}$ of the magnetic vector potential grow along with $A_{3}$ to cancel part of the poloidal field associated with $A_{3}$. Neither the perturbed $\psi$ relative to the equilibrium value nor the helical flux in this case are a measure of how much the magnetic topology is affected.

Instead we measure the island widths 'by hand' from Poincaré plots, the result of which is shown in Fig. 11 as a function of the quartic root of $E_{M \perp, n=1}=\frac{1}{2} \int\left(B_{R, n=1}^{2}+B_{Z, n=1}^{2}\right) d V$, the perturbed magnetic energy in the poloidal magnetic field. In reduced MHD, the island width is proportional to the square root of $\psi^{*}$ so that the magnetic energy, which is proportional to the square of $\psi^{*}$, is proportional to the fourth power of the island width. From Fig. 11 the resulting scaling can be seen to hold well also in full MHD. Therefore we can use the quartic root of the normalized magnetic energy in the poloidal magnetic field as a measure of the island width. Since the magnetic energy is proportional to the square of the magnetic field, the magnetic energy initially increases with twice the linear growth rate. Actually this is the way that growth rates are typically calculated in the current method:

$$
E \sim e^{2 \gamma t} \rightarrow \gamma=\frac{\ln \left(E_{1} / E_{0}\right)}{2\left(t_{1}-t_{0}\right)},
$$

where for the energy $E$ any quadratic quantity of a Fourier harmonic can be used. Using for example the kinetic energy $E_{k}=\frac{1}{2} \int \rho v^{2} d V$ or the magnetic energy $E_{M}=\frac{1}{2} \int B^{2} d V$ gives the same linear growth rate.

We follow the evolution of the tearing mode into the nonlinear regime using $N_{\psi}=80, N_{\vartheta}=40$ for the full MHD simulations and $N_{\psi}=101, N_{\vartheta}=61$ for the reduced MHD model. The island width as a function of time is shown in Fig. 12 . The island width initially grows with half the linear growth rate $\gamma$, as is shown in Fig. 12. After some time this exponential growth stalls and the island width oscillates with a small amplitude around its saturated size. There does not seem to be a clear region in which the island width grows linearly in time, as in the Rutherford regime. We think that for this test case saturation occurs at a value of $w$, which is too large to see the linear regime. 


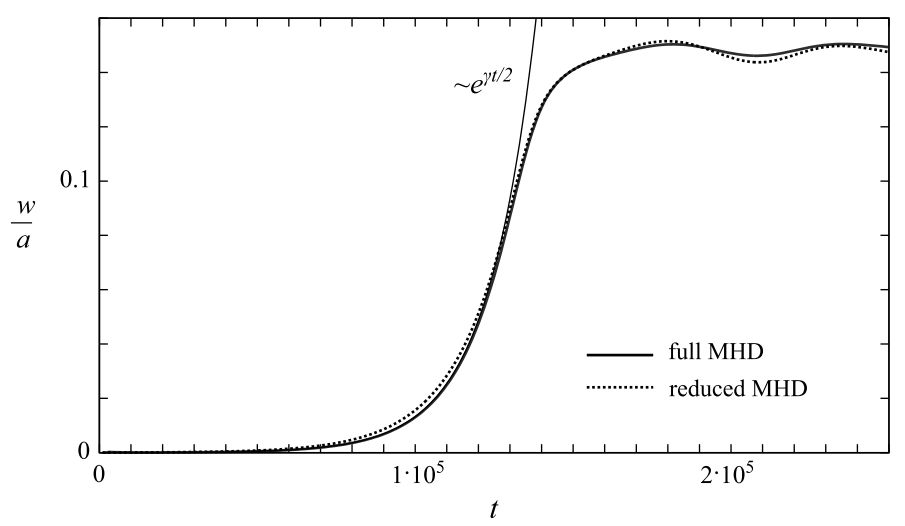

Fig. 12. The normalized island width $w / a$ as a function of time for the current full and a reduced MHD method including a toroidal velocity.

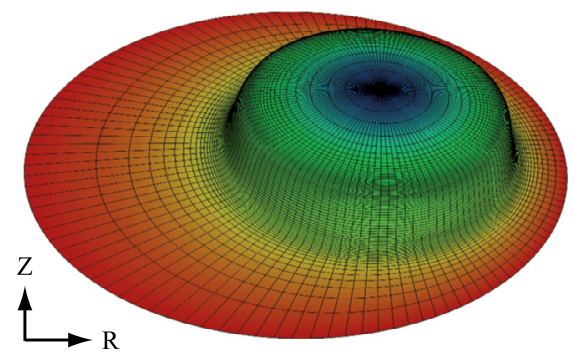

Fig. 13. A typical grid with radial grid accumulation. The height profile gives the equilibrium temperature, while the coloring denotes the equilibrium $\psi$.

The saturation phase is particularly challenging to simulate with the full MHD model. The small or negative tearing mode growth rates in this phase allow the growth of an instability on the grid axis associated with the violation of regularity discussed in Appendix A. To suppress this numerical instability we restrain also the coefficients of the second and fourth basis functions $b_{1,2}$ and $b_{1,4}$ on the axis to deviate from zero. The resulting decrease in the local order of accuracy on the axis is compensated by a local refinement of the elements near the axis, leading to a stable and accurate solution.

In these simulations we allow the axisymmetric component of all variables to evolve in time. To counteract resistive diffusion, the equilibrium current is subtracted from the current in the resistive term. Most of the $n=0$ variables do not significantly change in time now. There is a clear flattening of the temperature and density profiles though. This is expected as a consequence of the changed magnetic topology. The resulting flattening of the current density profile causes the growth of the tearing mode to saturate.

\subsection{Ballooning modes}

As a final illustration we take a first look at the ballooning mode instability. A standardized set of equilibria to study these instabilities was developed by Snyder. Here we use the cbm18_dens8 equilibrium used for benchmarking NIMROD [39], M3D- $C^{1}[40]$, and BOUT++ $[4,24]$ against various linear stability codes.

We leave a full benchmark and a comparison with the reduced MHD model for future work, and present here the result for a single toroidal mode number $n=12$.

The equilibrium has an ideally conducting circular wall with an aspect ratio $\epsilon^{-1}=3 / 2$. The density is constant, but the temperature profile has a strong H-mode-like pedestal gradient in a narrow layer. This profile is shown as an elevation in Fig. 13. The coloring denotes the equilibrium poloidal magnetic flux $\psi$ and a typical computational mesh is shown. The peak value of $\beta$ is approximately one percent. The safety factor profile ranges from around one on the axis, to between two and three in the pedestal region, while continuing to grow to large values outside this region.

The large pressure gradient in combination with unfavorable magnetic field curvature at the low field side is assumed to lead to an instability in which the plasma 'balloons' outwards; a topic for future research. Many poloidal harmonics that are resonant on different rational $q$-values overlap to form structures that are dominant on the low-field side. The total perturbed $n=12$ velocity and magnetic field are depicted in Fig. 14, showing the ballooning character of the mode and the strong radial and poloidal localization. Note how the structures get much more narrow near the high-field side where the local magnetic shear is higher.

Fig. 15 shows the perturbed density and plasma velocity at the low field side, revealing vortical structures of plasma motion. Note however the net plasma motion to the left of the figure, the top of the plasma. Because of symmetry in the 

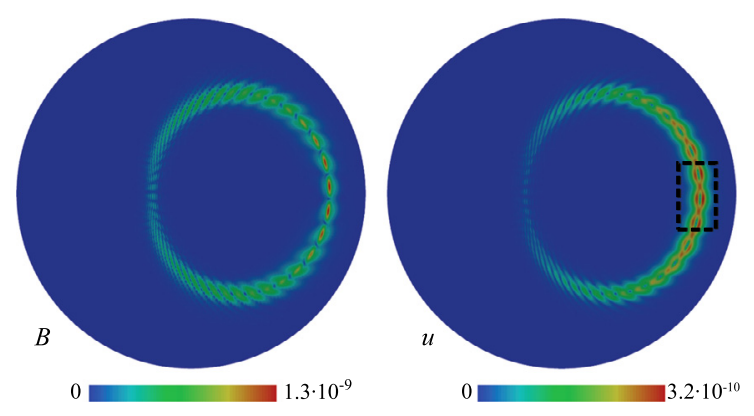

Fig. 14. The magnitude of the perturbed $n=12$ magnetic field (left) and velocity (right). The dashed box indicates the region shown in Fig. 15 .

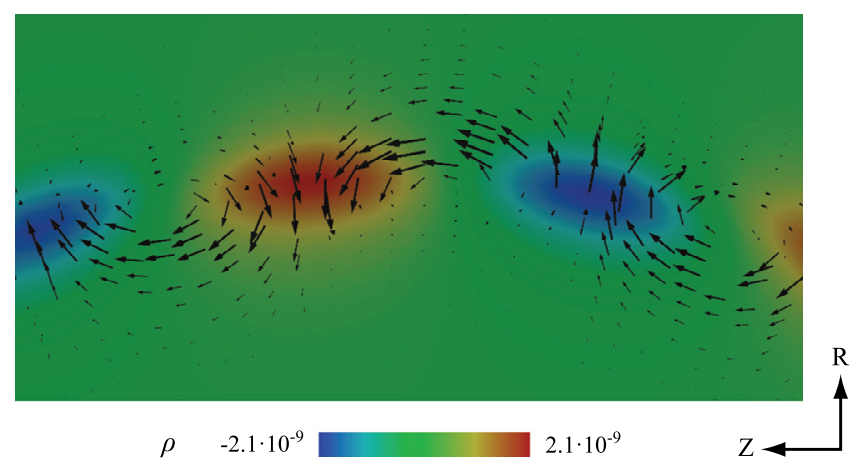

Fig. 15. The perturbed $n=12$ density at the low-field side together with the perturbed plasma velocity showing vortical structures with the same poloidal periodicity. See Fig. 14 for an indication of the location of the shown section.

top-bottom direction there should be an eigenmode with the exact same growth rate but with a net plasma motion towards the bottom of the plasma.

As was shown in [39] the linear instabilities of this equilibrium experience a significant kink drive, particularly impacting the smallest $n$-modes. The combination of external kink / peeling and ballooning effects is at the basis of Type- 1 ELMs.

\section{Conclusion}

We presented our extension to full MHD of the reduced MHD methods implemented in JOREK. To ensure a divergencefree magnetic field we deploy a vector potential formulation. The resulting gauge freedom is used to set the electrostatic potential to zero. Small noise in the components of the magnetic vector potential is found to pollute the solutions of linear MHD instabilities. We showed that this problem can be solved by projecting the momentum equation in a direction parallel to the magnetic field instead of in the toroidal direction.

The use of a structured rectangular mesh in a circular domain was shown to lead to an underdetermined system of equations. Additional conditions have to be supplied to ensure regularity of the solutions at the center of the grid. We derived conditions that can be imposed on the coefficients of the basis functions to ensure continuity of the solutions.

Various test cases based on analytical solutions were constructed to verify the implementation of the individual equations. Test cases with highly anisotropic diffusion showed that the same convergence as for isotropic diffusion can be obtained with our implementation. This surprising feat does not require the grid to be aligned with the magnetic field, as long as the grid is of good quality. This can be achieved by constructing the grid analytically or by accurate interpolation. The description of the magnetic field in terms of a stream function was shown to be essential for this property.

The complete system of full MHD equations was used to study what are the arguably three most important MHD instabilities in a tokamak: an internal kink mode, a tearing mode, and a ballooning mode. Their growth rates were compared with the result from linear stability codes and showed good agreement. A nice feature of our full MHD method is that it does not necessarily require a source of physical dissipation. Ballooning modes are driven by large pressure gradients and as such have a finite extended structure. These modes have been simulated without any resistivity, viscosity, or other physical diffusivity.

The tearing mode simulations were extended into the nonlinear regime and an insightful comparison was made with the reduced MHD models. For zero pressure, the reduced MHD models were found to be accurate in reproducing the full MHD growth rates. However, the inclusion of a toroidal velocity component was required, to approach the full MHD growth rates when including a finite pressure. Only minor differences between the two models remained in this case.

By and large these tearing mode simulations confirm what has been long known from research, namely that the reduced MHD model can give a reasonably accurate description of the predictions of the full MHD model. Agreement at nonzero 
pressure however requires a reduced MHD model that also includes a toroidal velocity component. For other phenomena the differences between the two models can be much larger. With the present extension of JOREK to the full MHD model we now have the possibility to easily verify this in the future.

\section{Acknowledgements}

This work, supported by NWO and the European Communities under the contract of the Association Euratom/FOM, was carried out within the framework of the European Fusion Program. The views and opinions expressed herein do not necessarily reflect those of the European Commission nor those of the ITER Organization. Part of this work was carried out using the HELIOS supercomputer system at the Computational Simulation Centre of the International Fusion Energy Research Centre (IFERC-CSC), Aomori, Japan, under the Broader Approach collaboration between Euratom and Japan, implemented by Fusion for Energy and JAEA. Part of this work was carried out using the HPC-FF cluster of the Jülich Supercomputing Centre, Germany.

Emiel van der Plas is gratefully acknowledged for his foundational work in implementing the full-MHD equations in JOREK. We thank Boniface Nkonga for his helpful comments on a previous version of this manuscript.

\section{Appendix A. Grid axis}

Near the axis of the grid, the quadrilateral elements are deformed into triangular elements. At the grid axis, there will typically be much more than the usual four nodes per vertex. Therefore, there will be more unknowns than equations, rendering the system of equations underdetermined. The corresponding freedom in the solutions may give rise to numerical instability, starting with oscillations near the axis. The solution is to either increase the number of equations, or to decrease the number of degrees of freedom. In principle, the same issue also holds for X-point grids, where six elements come together in a single point.

Additional equations can be obtained by imposing regularity of the solution at the axis. Expanding a continuous function $f(R, Z)$ to first-order in the distance to the axis at $R=R_{0}$ and $Z=Z_{0}$ gives

$$
f(R, Z) \approx f\left(R_{0}, Z_{0}\right)+\frac{\partial f}{\partial R}\left(R-R_{0}\right)+\frac{\partial f}{\partial Z}\left(Z-Z_{0}\right) .
$$

The scalar function $f$ can be any of our primitive variables (20). Introducing a polar coordinate system $R=R_{0}+r \cos \theta$, $Z=Z_{0}+r \sin \theta$ we have for $r \rightarrow 0$ :

$$
\begin{aligned}
\frac{\partial f}{\partial \theta} & =0, \\
\frac{\partial f}{\partial r} & =\frac{\partial f}{\partial R} \cos \theta+\frac{\partial f}{\partial Z} \sin \theta, \\
\frac{\partial^{2} f}{\partial r \partial \theta} & =-\frac{\partial f}{\partial R} \sin \theta+\frac{\partial f}{\partial Z} \cos \theta .
\end{aligned}
$$

The first of these equations shows the trivial fact that functions do not vary with $\theta$ at the single point in space corresponding to $r=0$. Eq. (A.2b) shows that for $r \rightarrow 0$ the radial derivative is only nonzero for functions with periodicity $2 \pi$. For such functions, $\partial f / \partial r$ changes sign when rotating over an angle $\pi$.

The regularity conditions (A.2a)-(A.2c) can provide the additional constraints required to reduce the redundancy in the degrees of freedom on the grid axis. Eq. (A.2a) for example excludes the use of the third basis function $b_{1,3}$ and $b_{4,3}$ for vertices 1 and 4 , of the elements near the axis. This is because $\partial b_{1,3} / \partial t$ and $\partial b_{4,3} / \partial t$ do not vanish for $s \rightarrow 0$. Since the coordinates $s$ and $t$ become locally proportional to $r$ and $\theta$ respectively, these basis functions therefore violate Eq. (A.2a). Writing down Eq. (A.2b) and (A.2c) for two values of $\theta$, we can solve for

$$
\begin{gathered}
\frac{\partial f}{\partial R}=\left.\frac{\partial f}{\partial r}\right|_{\theta_{1}} \cos \theta_{1}-\left.\frac{\partial^{2} f}{\partial r \partial \theta}\right|_{\theta_{1}} \sin \theta_{1}=\left.\frac{\partial f}{\partial r}\right|_{\theta_{2}} \cos \theta_{2}-\left.\frac{\partial^{2} f}{\partial r \partial \theta}\right|_{\theta_{2}} \sin \theta_{2}, \\
\frac{\partial f}{\partial Z}=\left.\frac{\partial f}{\partial r}\right|_{\theta_{1}} \sin \theta_{1}+\left.\frac{\partial^{2} f}{\partial r \partial \theta}\right|_{\theta_{1}} \cos \theta_{1}=\left.\frac{\partial f}{\partial r}\right|_{\theta_{2}} \sin \theta_{2}+\left.\frac{\partial^{2} f}{\partial r \partial \theta}\right|_{\theta_{2}} \cos \theta_{2} .
\end{gathered}
$$

For $s \rightarrow 0$ and $t=0$ we have $\partial^{2} b_{1,4} / \partial s \partial t=9$ and $\partial b_{1,2} / \partial s=3$. With $\theta_{1}$ and $\theta_{2}$ the values of $\theta$ for two consecutive axis nodes, Eqs. (A.3) give additional conditions for the coefficients of these basis functions.

Instead of adding these conditions as additional equations, we can also impose them on the basis functions themselves. We then look for basis functions that are local to all the elements near the axis and satisfy the above regularity conditions. Additionally they should allow for continuity of the variables along element boundaries, and preferably also be exactly integrable with the Gauss quadrature presently used. Obvious candidates are linear combinations of our basis functions. A first one, $b_{a x, 1}$ is easy. The sum of all $b_{1,1}$ and $b_{4,1}$ of the elements that border the axis satisfies these conditions. A second basis function follows from Eq. (A.3a) as 

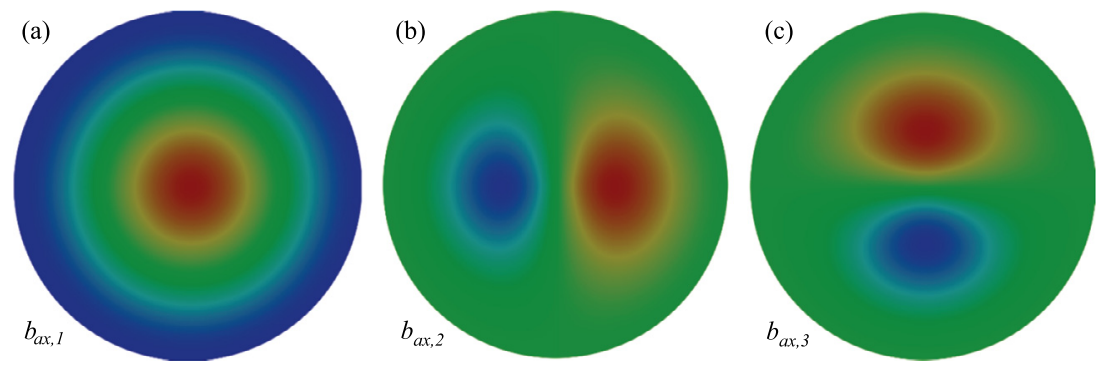

Fig. A.16. The three basis functions that satisfy the regularity conditions at the axis describe: (a) the function value $f$, (b) $\partial f / \partial R$, and (c) $\partial f / \partial Z$ at the axis.

$$
b_{a x, 2}=\cos \theta_{1} b_{1,2}-\frac{1}{3} \sin \theta_{1} b_{1,4}+\cos \theta_{2} b_{4,2}+\frac{1}{3} \sin \theta_{2} b_{4,4} .
$$

A third one follows from (A.3b) as

$$
b_{a x, 3}=\sin \theta_{1} b_{1,2}+\frac{1}{3} \cos \theta_{1} b_{1,4}+\sin \theta_{2} b_{4,2}-\frac{1}{3} \cos \theta_{2} b_{4,4} .
$$

These three basis functions are shown in Fig. A.16. The coefficients of these basis functions represent the respective values of $f,(\partial f / \partial R)(\partial r / \partial s)$, and $(\partial f / \partial Z)(\partial r / \partial s)$ at the axis. Using these modified axis basis functions, the regularity conditions are automatically satisfied, and $C^{1}$ continuity at the axis is guaranteed. Note that the basis function $b_{a x, 3}$ is equal to $b_{a x, 2}$ when rotated over $\pi / 2$. As such, taking a linear combination of these two new basis functions amounts to a rotation and scaling.

What is being done to ensure unique function values at the axis is: we make the coefficients of the first basis functions of all axis nodes equal. This is equivalent to introducing the single axis basis function of Fig. A.16. This already reduces a lot of the redundancy in the degrees of freedom and is often enough to ensure stable and accurate solutions. However, the coefficients of the $b_{1,3}$ and $b_{4,3}$ basis functions may still become significant. Therefore, in the following, we effectively remove these inadmissible basis functions by forcing their coefficients to be zero. For the simulation of the tearing mode saturation phase we furthermore constrain the coefficients of the $b_{1,2}$ and $b_{1,4}$ basis functions to zero on the axis.

Note that this locally reduces the order of convergence. Probably because of the typically mild variation of the solutions near the grid axis and the relatively small element sizes near the grid axis, this does not adversely impact the error in the test cases considered in this paper. Therefore we implement the other two axis basis functions $b_{a x, 2}$ and $b_{a x, 3}$, shown in Fig. A.16(b) and (c) respectively. If regularity at the axis is an issue, then the solution proposed in this section can be fully implemented.

\section{References}

[1] C.R. Sovinec, A.H. Glasser, T.A. Gianakon, D.C. Barnes, R.A. Nebel, et al., Nonlinear magnetohydrodynamics simulations using high-order finite elements, J. Comput. Phys. 195 (2004) 355-386.

[2] C.R. Sovinec, J.R. King, the NIMROD Team, Analysis of a mixed semi-implicit/implicit algorithm for low-frequency two-fluid plasma modeling, J. Comput. Phys. 229 (2010) 5803-5819.

[3] B.D. Dudson, M.V. Umansky, X.Q. Xu, P.B. Snyder, H.R. Wilson, BOUT++: a framework for parallel plasma fluid simulations, Comput. Phys. Commun. 180 (2009) 1467-1480.

[4] B.D. Dudson, X.Q. Xu, M.V. Umansky, H.R. Wilson, P.B. Snyder, Simulation of edge localized modes using BOUT++, Plasma Phys. Control. Fusion 53 (2011) 054005.

[5] N.M. Ferraro, S.C. Jardin, Calculations of two-fluid magnetohydrodynamic axisymmetric steady-states, J. Comput. Phys. 228 (2009) $7742-7770$.

[6] J. Breslau, N. Ferraro, S. Jardin, Some properties of the M3D- $C^{1}$ form of the three-dimensional magnetohydrodynamics equations, Phys. Plasmas 16 (2009) 092503.

[7] H. Lütjens, J.-F. Luciani, The XTOR code for nonlinear 3D simulations of MHD instabilities in tokamak plasmas, J. Comput. Phys. 227 (2008) $6944-6966$.

[8] H. Lütjens, J.-F. Luciani, XTOR-2F: a fully implicit Newton-Krylov solver applied to nonlinear 3D extended MHD in tokamaks, J. Comput. Phys. 229 (2010) 8130-8143.

[9] L.A. Charlton, J.A. Holmes, H.R. Hicks, V.E. Lynch, B.A. Carreras, Numerical calculations using the full MHD equations in toroidal geometry, J. Comput. Phys. 63 (1986) 107-129.

[10] Y. Todo, K. Shinohara, M. Takechi, M. Ishikawa, Nonlocal energetic particle mode in a JT-60U plasma, Phys. Plasmas 12 (2005) 012503.

[11] G.T.A. Huysmans, External kink (peeling) modes in x-point geometry, Plasma Phys. Control. Fusion 47 (2005) 2107-2121.

[12] G.T.A. Huysmans, ELMs: MHD instabilities at the transport barrier, Plasma Phys. Control. Fusion 47 (2005) B165-B178.

[13] G.T.A. Huysmans, MHD stability in X-point geometry: simulation of ELMs, Nucl. Fusion 47 (2007) 659-666.

[14] G.T.A. Huysmans, S. Pamela, E. van der Plas, P. Ramet, Non-linear MHD simulations of edge localized modes (ELMs), Plasma Phys. Control. Fusion 51 (2009) 124012.

[15] S.J.P. Pamela, G.T.A. Huysmans, M.N.A. Beurskens, S. Devaux, T. Eich, et al., Nonlinear MHD simulations of edge-localized-modes in JET, Plasma Phys. Control. Fusion 53 (5) (2011) 054014.

[16] M. Hölzl, S. Günter, R.P. Wenninger, W.C. Müller, G.T.A. Huysmans, et al., Reduced-magnetohydrodynamic simulations of toroidally and poloidally localized edge localized modes, Phys. Plasmas 19 (8) (2012) 082505.

[17] S. Pamela, G. Huysmans, S. Benkadda, Influence of poloidal equilibrium rotation in MHD simulations of edge-localized modes, Plasma Phys. Control. Fusion 52 (7) (2010) 075006. 
[18] E. Nardon, M. Bécoulet, G. Huysmans, O. Czarny, Magnetohydrodynamics modelling of H-mode plasma response to external resonant magnetic perturbations, Phys. Plasmas 14 (2007) 092501.

[19] F. Orain, M. Bécoulet, G. Huysmans, G. Dif-Pradalier, V. Grandgirard, et al., Interaction of resonant magnetic perturbations with flows in toroidal geometry, in: 39th EPS Conference on Plasma Physics, vol. 4, 2012, p. 65.

[20] C. Reux, G. Huysmans, J. Bucalossi, M. Bécoulet, Non-linear simulations of disruption mitigation using massive gas injection on tore supra, in: 38th EPS Conf. Plasma Phys., vol. 3, 2011, p. 117.

[21] O. Czarny, G. Huysmans, Bézier surfaces and finite elements for MHD simulations, J. Comput. Phys. 227 (2008) 7423-7445.

[22] G.T.A. Huysmans, Implementation of an iterative solver in the non-linear MHD code JOREK, Technical report, ANR, 2006.

[23] E.M. Edlund, M. Porkolab, G.J. Kramer, L. Lin, Y. Lin, N. Tsujii, S.J. Wukitch, Experimental study of reversed shear Alfvén eigenmodes during the current ramp in the Alcator C-Mod tokamak, Plasma Phys. Control. Fusion 52 (2010) 115003.

[24] X.Q. Xu, B.D. Dudson, P.B. Snyder, M.V. Umansky, H.R. Wilson, T. Casper, Nonlinear ELM simulations based on a nonideal peeling-ballooning model using the BOUT++ code, Nucl. Fusion 51 (10) (2011) 103040.

[25] C. Helzel, J.A. Rossmanith, B. Taetz, An unstaggered constrained transport method for the 3D ideal magnetohydrodynamic equations, J. Comput. Phys. 230 (2011) 3803-3829.

[26] G.T.A. Huysmans, R. Abgrall, M. Bécoulet, R. Huart, B. Nkonga, et al., Non-linear MHD simulations of ELMs, in: 35rd EPS Conference on Plasma Physics, vol. 32D, 2008, p. 2.065.

[27] M. Hölzl, P. Merkel, G.T.A. Huysmans, E. Nardon, E. Strumberger, R. McAdams, I. Chapman, S. Günter, K. Lackner, Coupling JOREK and STARWALL codes for non-linear resistive-wall simulations, J. Phys. 401 (2012) 012010.

[28] J.W. Haverkort, Magnetohydrodynamic waves and instabilities in rotating tokamak plasmas, PhD thesis, Eindhoven University of Technology, 2013, http://repository.tue.nl/750676.

[29] J.P. Goedbloed, S. Poedts, Principles of Magnetohydrodynamics, Cambridge University Press, 2004.

[30] J.W. Haverkort, H.J. de Blank, B. Koren, The Brunt-Väisälä frequency of rotating tokamak plasmas, J. Comput. Phys. 231 (2012) 981-1001.

[31] A.J.C. Beliën, M.A. Botchev, J.P. Goedbloed, B. van der Holst, R. Keppens, FINESSE: axisymmetric MHD equilibria with flow, J. Comput. Phys. 11 (182) (2002) $91-117$.

[32] E. Hairer, G. Wanner, Solving Ordinary Differential Equations II: Stiff and Differential-Algebraic Problems, Springer, 1996.

[33] P. Hénon, P. Ramet, J. Roman, On finding approximate supernodes for an efficient block-ILU(k) factorization, Parallel Comput. 34 (6) (2008) $345-362$.

[34] R.M. Beam, R.F. Warming, Alternating direction implicit methods for parabolic equations with a mixed derivative, SIAM J. Sci. Stat. Comput. 1 (1) (1980) $131-159$.

[35] G.G. Dahlquist, A special stability problem for linear multistep methods, BIT Numer. Math. 3 (1963) 27-43.

[36] A. Chupin, R. Stepanov, Full perturbation solution for the flow in a rotating torus, Phys. Rev. E 77 (2008) 057301.

[37] J.P. Goedbloed, A.J.C. Beliën, B. van der Holst, R. Keppens, Unstable continuous spectra of transonic axisymmetric plasmas, Phys. Plasmas 11 (2004) $28-54$.

[38] J.W.S. Blokland, B. van der Holst, R. Keppens, J.P. Goedbloed, PHOENIX: MHD spectral code for rotating laboratory and gravitating astrophysical plasmas, J. Comput. Phys. 226 (2007) 509-533.

[39] B.J. Burke, S.E. Kruger, C.C. Hegna, P. Zhu, P.B. Snyder, C.R. Sovinec, E.C. Howell, Edge localized linear ideal magnetohydrodynamic instability studies in an extended-magnetohydrodynamic code, Phys. Plasmas 17 (2010) 032103.

[40] N.M. Ferraro, S.C. Jardin, P.B. Snyder, Ideal and resistive edge stability calculations with M3D-C ${ }^{1}$, Phys. Plasmas 17 (2010) 102508. 